ARTICLE

Received 4 Jun 2014 | Accepted 16 Feb 2015 | Published 26 Mar 2015

DOI: $10.1038 /$ ncomms7653

\title{
The transcription factor Foxc1 is necessary for Ihh-Gli2-regulated endochondral ossification
}

Michiko Yoshida ${ }^{1,2,}$, Kenji Hata ${ }^{1, \star}$, Rikako Takashima ${ }^{1}$ Koichiro Ono ${ }^{1}$, Eriko Nakamura ${ }^{1}$, Yoshifumi Takahata ${ }^{1}$ Tomohiko Murakami ${ }^{1}$, Sachiko Iseki ${ }^{3}$, Teruko Takano-Yamamoto ${ }^{2}$, Riko Nishimura ${ }^{1} \&$ Toshiyuki Yoneda ${ }^{1,4}$

Indian hedgehog (Ihh) regulates endochondral ossification in both a parathyroid hormonerelated protein (PTHrP)-dependent and -independent manner by activating transcriptional mediator Gli2. However, the molecular mechanisms underlying these processes remain elusive. Here by using in vivo microarray analysis, we identify forkhead box C1 (Foxc1) as a transcriptional partner of Gli2. Foxc1 stimulates expression of Ihh target genes, including PTHrP and Col10a1, through its physical and functional interaction with Gli2. Conversely, a dominant negative Foxc1 inhibits the Ihh target gene expression. In a spontaneous loss of Foxc1 function mouse (Foxc1 ${ }^{\text {ch/ch}}$ ), endochondral ossification is delayed and the expression of Ihh target genes inhibited. Moreover, the pathological Foxc1 missense mutation observed in the Axenfeld-Rieger syndrome impairs Gli2-Foxc1 association as well as Ihh function. Our findings suggest that Foxc1 is an important transcriptional partner of Ihh-Gli2 signalling during endochondral ossification, and that disruption of the Foxc1-Gli2 interaction causes skeletal abnormalities observed in the Axenfeld-Rieger syndrome.

\footnotetext{
${ }^{1}$ Department of Molecular and Cellular Biochemistry, Osaka University Graduate School of Dentistry, 1-8 Yamadaoka, Suita 565-0871, Japan. ${ }^{2}$ Division of Orthodontics and Dentofacial Orthopedics, Tohoku University Graduate School of Dentistry, 4-1 Seiryo-machi, Aoba-ku, Sendai 980-8575, Japan. ${ }^{3}$ Section of Molecular Craniofacial Embryology, Graduate School of Medical and Dental Sciences, Tokyo Medical and Dental University, 1-5-45 Yushima, Bunkyo-ku, Tokyo 113-8549, Japan. ${ }^{4}$ Division of Hematology/Oncology, Indiana University School of Medicine, Walther Hall, R3-C321D, 980 West Walnut Street, Indianapolis, Indiana 46202, USA. * These authors contributed equally to this work. Correspondence and requests for materials should be addressed to K.H. (email: hata@dent.osaka-u.ac.jp) or to R.N. (email: rikonisi@dent.osaka-u.ac.jp).
} 
S keletal development occurs via two independent biological processes known as intramembranous ossification and endochondral ossification ${ }^{1}$. During intramembranous ossification, mesenchymal stem cells differentiate into osteoblasts that produce and mineralize bone matrix without cartilage template. The clavicle and flat bones, including calvaria, mandible and maxilla, are formed by intramembranous ossification $^{2,3}$. In contrast, endochondral ossification is mainly conducted by chondrocytes and involves sequential steps of chondrocyte differentiation followed by the replacement of bone $\mathrm{e}^{4}$. During early stage chondrocyte differentiation, mesenchymal cells condense and differentiate into resting and proliferating chondrocytes that produce abundant extracellular matrix, including type- 2 collagen and aggrecan ${ }^{5}$. In the late stage, proliferating chondrocytes become hypertrophic chondrocytes that produce type-10 collagen and matrix metalloproteinase (MMP)-13 (ref. 4). Finally, the hypertrophic chondrocytes undergo apoptosis, which is followed by the invasion of blood vessels and osteoblasts ${ }^{6}$. In humans, most long bones and some craniofacial bones, including the mandibular condyle and sphenoid bone, are formed by endochondral ossification. Thus, the impairment of endochondral ossification causes various skeletal abnormalities, including a short stature and facial dysmorphism ${ }^{7}$.

Different cytokines, growth factors and hormones regulate endochondral ossification by activating intracellular signalling molecules and transcription factors. Bone morphogenetic protein family members control chondrocyte proliferation and matrix synthesis by activating Smads ${ }^{8,9}$; cartilage-specific deletion of Smad1/5 in mice results in severe chondrodysplasia ${ }^{10}$. Indian hedgehog (Ihh), a member of the hedgehog family, plays critical roles in the endochondral ossification via its regulation of genes, such as parathyroid hormone-related protein $(P T H r P)$, protein patched homologue 1 (Ptch1), $\alpha 1(\mathrm{X})$ collagen (Col10a1) and Gli1, by activating the transcription factor Gli2 (refs 11-14). The deletion of Ihh or Gli2 causes severe skeletal defects with reduced chondrocyte proliferation and maturation ${ }^{15-17}$. In addition, Sexdetermining region Y-type high mobility group box protein 9 (Sox9) and Runt-related transcription factor $2 / 3$ (Runx2/3), are critical transcription factors for early chondrogenesis ${ }^{18}$ and chondrocyte hypertrophy ${ }^{19}$.

The transcriptional machineries of individual genes assemble into transcriptional networks that orchestrate the complicated but well-organized chondrocyte differentiation steps during endochondral ossification ${ }^{20}$. Thus, the identification and characterization of novel transcription factors involved in these processes will contribute to a better understanding of endochondral ossification. Numerous attempts have been made to identify these and we previously identified several transcription factors using a high-throughput assay system ${ }^{21-24}$ and Solexa deep-sequencing technology ${ }^{25}$. However, the transcriptional networks of endochondral ossification are still not fully elucidated.

In this study, we established a gene-screening system to identify novel transcription factors involved in endochondral ossification using a combination of chondrocyte reporter mice and flow cytometry (fluorescence-activated cell sorting, FACS)assisted microarray analysis. This revealed that forkhead box $\mathrm{cl}$ (Foxc1) plays a critical role in endochondral ossification through its physical and functional association with Ihh-Gli2 signalling. We also established that the loss of interaction caused by a missense mutation in Foxc1 partially accounts for pathogenesis of the abnormal skeletal development observed in the AxenfeldRieger syndrome. Our findings provide new insights into the transcriptional network system that is critical for endochondral ossification and demonstrate a pathogenic mechanism of skeletal abnormalities in the Axenfeld-Rieger syndrome.

\section{Results}

Identification of genes selectively expressed in cartilage. We first attempted to identify transcription factors that were highly expressed in developing cartilage during the embryonic stage. In particular, we aimed to isolate transcription factors relevant to the in vivo situation. We, therefore, generated transgenic mice in which the chondrocytes were fluorescently labelled with the Venus gene expression reporter driven by the Col2al promoter (Col2a1-Venus-Tg; Supplementary Fig. 1a). Exogenous expression of Venus in cartilage tissues of Col2a1Venus-Tg mice was confirmed by fluorescence stereomicroscopy (Supplementary Fig. 1b,c). Cartilage from the limbs, rib cages and vertebrae of E13.5 Col2a1-Venus-Tg mice was dissociated and digested into single cells using collagenase and trypsin (Supplementary Fig. 1d).

Venus-negative and -positive cells were sorted using FACS Aria, and RNA was isolated from each population without cell expansion (Supplementary Fig. 1d). Reverse transcription polymerase chain reaction (RT-PCR) analysis demonstrated that the Venus gene was only expressed in Venus-positive cells (Supplementary Fig. 1e). We purified $2.0 \mu \mathrm{g}$ total RNA from Venus-negative cells and $1.75 \mu \mathrm{g}$ total RNA from Venus-positive cells and subjected it to microarray analysis using an Affymetrix Mouse Genome 430 2.0 Array. High chondrocyte gene expression was observed in Venus-positive cells by quantitative RT-PCR (RT-qPCR; Fig. 1a) and microarray analysis (Supplementary Table 1), indicating that purification of chondrocytes and gene expression profiling was successful. Microarray analysis, focusing on transcription factors, also showed that Sox5, Sox6 and Sox9 were highly expressed in Venus-positive cells (Table 1). From the 10 most highly expressed transcription factors shown in Table 1, we selected Foxc1 as a suitable candidate because human FOXC1 mutations cause the Axenfeld-Rieger syndrome, which is characterized by skeletal abnormalities ${ }^{26,27}$. However, the role of Foxc1 in chondrogenesis is unknown. Venus-positive cells expressed a high level of Foxc1, (1284) similar to that for Sox9 (1601; Table 1) and Foxc1 expression was increased by 7.03 -fold compared with Venus-negative cells (Fig. 1b).

Expression of Foxc1 in chondrocytes. We next used RT-qPCR to demonstrate high levels of Foxc1 expression in the sternum and rib cartilage, where Col2a1 is also highly expressed (Fig. 1c). Whole-mount in situ hybridization analysis of E12.5 mice revealed high Foxc1 expression in developing limb buds (Fig. 1d) and in situ hybridization analysis of E15.5 mouse tibias showed strong expression of Foxcl in Col2a1-positive chondrocytes (Fig. 1e). Importantly, Foxc1 mRNA was detected in distal chondrocytes, which are known to produce PTHrP (Fig. 1e). Immunohistochemical analysis of E13.5 developing limb buds also demonstrated that Foxcl was detected not only in proliferating chondrocytes but also in perichondrial cells at the end of growth plates where PTHrP is synthesized (Supplementary Fig. 2a). Moreover, Foxc1 was expressed in resting, proliferating and hypertrophic chondrocytes in the tibial growth plates of newborn mice (Supplementary Fig. 2b). These data collectively suggest that Foxcl might play multifunctional roles during both early and late endochondral ossification.

Foxc1 is critical for endochondral ossification in vivo. Using a spontaneous loss of Foxc1 function mutant mouse $\left(\right.$ Foxc1 $\left.{ }^{\text {ch/ch }}\right)$, we determined the in vivo significance of Foxc1 during endochondral ossification. Foxc1 $1^{\text {ch/ch }}$ mice died soon after birth and showed abnormal skeletal development, as previously described $^{28}$. Skeletal preparations of newborn Foxc1 ${ }^{\text {ch/ch }}$ mice showed a reduction in Alcian Blue staining, no ossification centre in the 
a
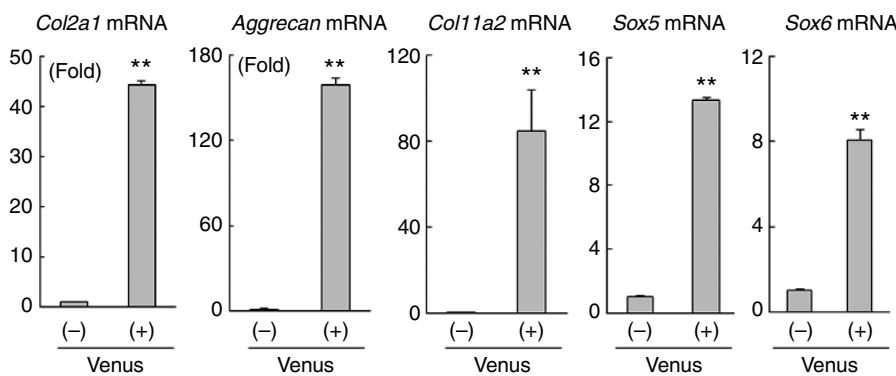

Sox9 mRNA

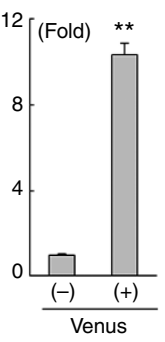

b

C
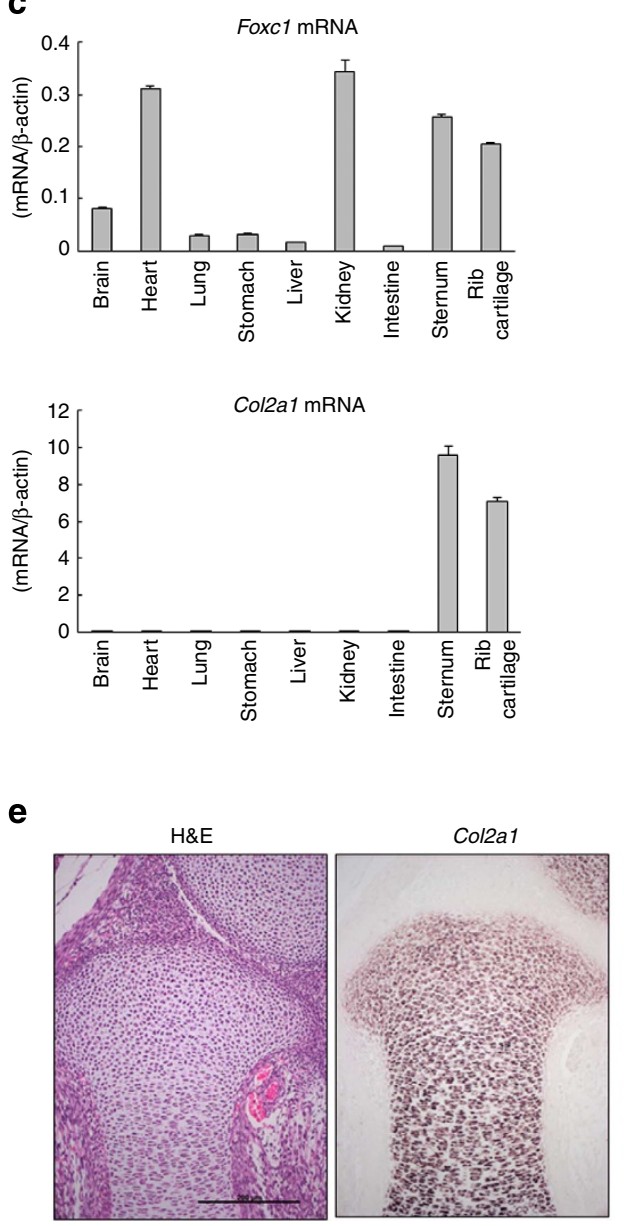

d
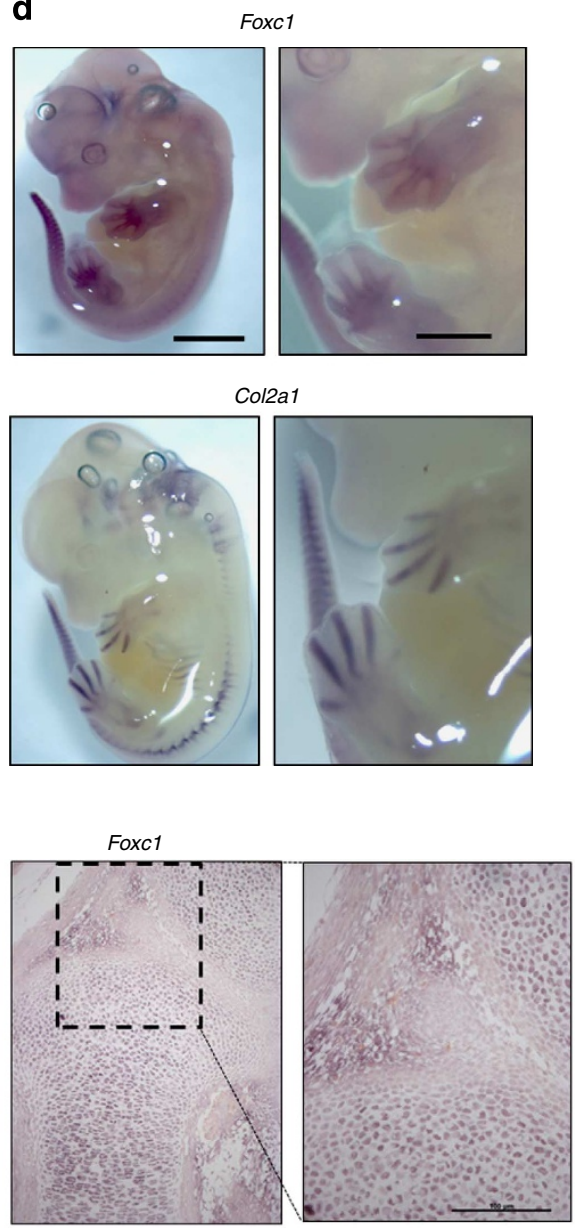

Figure 1 | Identification of Foxc1 as a candidate transcription factor selectively expressed in chondrocytes. (a,b) Total RNA isolated from Venusnegative and -positive cells was analysed by RT-qPCR for chondrocyte marker genes (a) and Foxc1 (b) expression. Data are shown as the mean \pm s.d. $(n=3) .{ }^{* \star} P<0.01$ (versus Venus $(-)$ ); Student's $t$-test. (c) Tissue distribution of Foxc1 (upper panel) and Col2al (lower panel) mRNA in newborn mouse tissues. Total RNA isolated from indicated tissues was analysed by RT-qPCR. Data are shown as the mean \pm s.d. $(n=3)$. (d) The expression of Foxc1 and Col2a1 in developing limbs of E12.5 mice was determined by whole-mount in situ hybridization. Scale bar, 2 mm (left panel), $1 \mathrm{~mm}$ (right panel). (e) Haematoxylin and eosin (H\&E) staining and in situ hybridization analysis of Col2al (middle panel) and Foxc1 (right panel) in growth plate chondrocytes of E15.5 mice tibia. The boxed area shows higher magnification of distal chondrocytes. Scale bar, $200 \mu \mathrm{m}$ (lower magnification, $\times 20)$ and 100 $\mu \mathrm{m}$ (higher magnification, $\times 40$ ).

sternum and impaired calcification in vertebrae compared with wild-type (WT) mice (Supplementary Fig. 3a,b). Moreover, Foxc1 $1^{\text {ch/ch }}$ mice exhibited significantly shorter limbs than WT (Supplementary Fig. 3c-e). Consistent with this, histological analysis of femurs (Fig. 2a) and tibias (Supplementary Fig. 3f) of newborn mice also showed weaker Alcian Blue staining and a smaller ossification centre in Foxc $1^{\text {ch/ch }}$ mice compared with WT littermates. Notably, the sternum phenotype was characterized by disorganized rib fusion, the complete absence of hypertrophic chondrocytes and ossification (Fig. 2b). Further analysis of tibial growth plate chondrocytes revealed that the Foxc1 $1^{\text {ch/ch }}$ hypertrophic chondrocyte zone was significantly longer than that of WT mice (Fig. 2c,d). These data suggest that Foxc1 inactivation disrupts endochondral ossification, leading to an abnormal skeletal phenotype.

To further investigate endochondral ossification in $\mathrm{Foxcl}^{\mathrm{ch} / \mathrm{ch}}$ mice, we investigated embryonic growth plate chondrocytes. Foxc1 ${ }^{\text {ch/ch }}$ mice at E15.5 showed a slight dwarf phenotype and 


\begin{tabular}{|lllcc|}
\hline \multicolumn{3}{l}{ Table 1 | Ten most highly expressed transcription factors in } \\
Venus-positive cells.
\end{tabular}

smaller ossification areas (Supplementary Fig. 4a,b), and haematoxylin and eosin (H\&E) staining of E15.5 tibias demonstrated degradation of the hypertrophic chondrocyte zone in the centre of WT but not Foxc1 ${ }^{\text {ch/ch }}$ tibias (Fig. 2e). In situ hybridization analysis of E15.5 tibias revealed a reduced and delayed Col10a1-positive zone in Foxc1 $1^{\text {ch } / c h}$ mice compared with that of WT mice, whereas the expression patterns of other chondrocyte genes were unchanged (Fig. 2e). Immunofluorescence analysis also showed a decrease in the Col10-positive area and a delay of Collo expression in Foxc1 $1^{\text {ch/ch }}$ mice compared with that in WT mice (Supplementary Fig. 4c). Moreover, MMP13 expression was markedly diminished (Fig. 2f) and the invasion of Colla1- and Runx2-positive osteoblasts was not observed in Foxc1 ${ }^{\text {ch/ch }}$ mice (Supplementary Fig. 4d), suggesting retarded chondrocyte hypertrophy. Collectively, these data suggest that Foxc1 ${ }^{\text {ch/ch }}$ mice exhibit abnormal skeletal development because of a delay in endochondral ossification.

Foxcl directly regulates $\mathbf{P T H} \boldsymbol{P} \boldsymbol{P}$ expression. We next examined the functional role of Foxc1 in endochondral ossification. Because Foxc1 protein is expressed in resting, proliferating and hypertrophic chondrocytes (Supplementary Fig. 2b), we reasoned that it must be involved in both early and late stages of endochondral ossification. This idea is consistent with results observed in Foxc1 ${ }^{\text {ch/ch }}$ mice (Fig. 2a-c, Supplementary Fig. 3). To test this, we used RT-qPCR to identify Foxcl target genes in resting and proliferating chondrocytes. When Foxcl was overexpressed in primary chondrocytes, it markedly increased $P T H r P$ expression (Fig. 3a). Together with our earlier immunohistochemical analysis (Supplementary Fig. 2a), which showed the expression of Foxc1 in perichondrial cells at the end of growth plates, this suggests that $P T H r P$ is a target gene of Foxcl in the early stage of chondrocyte development. In support of this, the PTHrP promoter was found to contain a motif that fits the core consensus Foxc1-binding sequence ${ }^{29}$ (Fig. 3b). DNA pull-down assays using the biotinylated Foxc1-binding element demonstrated that Foxc1 directly bound to this element and that binding was markedly competed with by excess non-biotinylated probe in a dosedependent manner (Fig. 3b). Chromatin immunoprecipitation (ChIP) with an anti-Foxcl antibody showed the direct binding of Foxcl to the PTHrP promoter (Fig. 3c). Moreover, Foxc1 increased the promoter activity of a reporter construct containing six copies of the Foxcl-binding element from the $\mathrm{PTHrP}$ promoter in a dose-dependent manner (Fig. 3d). Foxc1 was further shown to regulate $P T H r P$ expression because mRNA isolated from Foxc1 $1^{\text {ch/ch }}$ limbs had reduced levels of PTHrP mRNA compared with that from WT limbs (Fig. 3e). Of note, a BrdU labelling assay demonstrated that Foxc1 $1^{c h / c h}$ chondrocytes had a significantly decreased proliferation rate compared with that of WT chondrocytes, suggesting that the Foxc1-dependent PTHrP action in growth plate chondrocytes was impaired in Foxc1 $1^{\text {ch/ch }}$ mice (Supplementary Fig. 5). Taken together, these data indicate that $\mathrm{PTHrP}$ is likely to be a direct target gene of Foxc1 in chondrocytes.

Foxc1 regulates Ihh target gene expression with Gli2. Accumulating evidence shows that Ihh and its major signalling molecule, Gli2, regulate PTHrP expression in chondrocytes ${ }^{15,30}$. For example, the targeted disruption of Gli2 in mice causes a reduction in PTHrP expression and abnormal endochondral ossification ${ }^{17}$. These reports led us to hypothesize that Foxc1 associates with Ihh-Gli2 signalling in the regulation of PTHrP. We observed no significant difference in Ihh mRNA levels

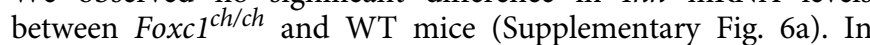
addition, the overexpression of Foxcl had no effect on Ihh mRNA levels in the presence or absence of Gli2 (Supplementary Fig. 6b). These data suggest that Foxcl does not affect Ihh expression. In contrast, the concomitant expression of Foxc1 and Ihh in primary chondrocytes increased PTHrP expression to levels higher than that induced by Ihh alone (Fig. 3f). These data suggest a functional cooperation between Foxc1 and intracellular signalling activated by Ihh. Notably, a synergistic effect between Foxc1 and Gli2 was observed to affect $P T H r P$ expression in primary chondrocytes (Fig. 3g), while a dominant negative (DN) Gli2 decreased Foxcl-dependent PTHrP expression (Fig. 3h). Moreover, immunoprecipitation (IP)-western analysis revealed that Foxc1 physically associates with Gli2 (Fig. 3i). In addition, red fluorescent protein (RFP)-tagged Foxc1 co-localized with Venus-tagged Gli2 in the nucleus (Fig. 3j). Collectively, these data suggest that Foxc1 regulates PTHrP expression through its functional and physical interaction with Ihh-Gli2 signalling.

In addition to $P T H r P$, Ihh-Gli2 signalling also directly controls the expression of several genes during endochondral ossification, including Gli1 and Ptch1 (ref. 12). Thus, we next determined whether Foxc1, with the involvement of Ihh-Gli2 signalling, modulates Gli1 and Ptch1 expression. Although Foxc1 alone failed to increase Gli1 and Ptch1 mRNA levels in primary chondrocytes, it could stimulate Gli1 and Ptch1 expression induced by Ihh (Fig. 4a,b). Consistent with the PTHrP mRNA induction (Fig. 3g), the concomitant overexpression of Foxc1 and Gli2 synergistically increased Gli1 and Ptch1 expression levels in primary chondrocytes (Fig. 4c,d). It should be noted that Gli1 and Ptch1 expression in Foxc1 $1^{\text {ch/ch }}$ tibias was reduced compared with that in WT mice (Fig. 4e,f). Moreover, Foxc1 increased the binding of Gli2 to the Gli-binding element located in the PTHrP, Gli1 (ref. 31) and Ptch1 (ref. 32) promoters, as shown by ChIP assays using an anti-Gli2 antibody (Fig. $4 \mathrm{~g}-\mathrm{i}$ ). Of note, the binding activity of endogenous Gli2 to the Gli-binding element located in the PTHrP, Gli1 and Ptch1 gene promoters was significantly decreased in Foxc1 $1^{\text {ch/ch }}$ chondrocytes compared with that in WT chondrocytes (Supplementary Fig. 7). These results collectively suggest that Foxc1, as a transcriptional partner of Gli2, promotes the expression of Ihh target genes, which, in turn, regulates endochondral ossification.

Col10a1 is a target gene of Foxc1 in chondrocytes. Foxc1 is expressed in hypertrophic chondrocytes (Supplementary Fig. 2b); therefore, we next examined its role in the late stage of endochondral ossification. We overexpressed Foxcl in primary 
a

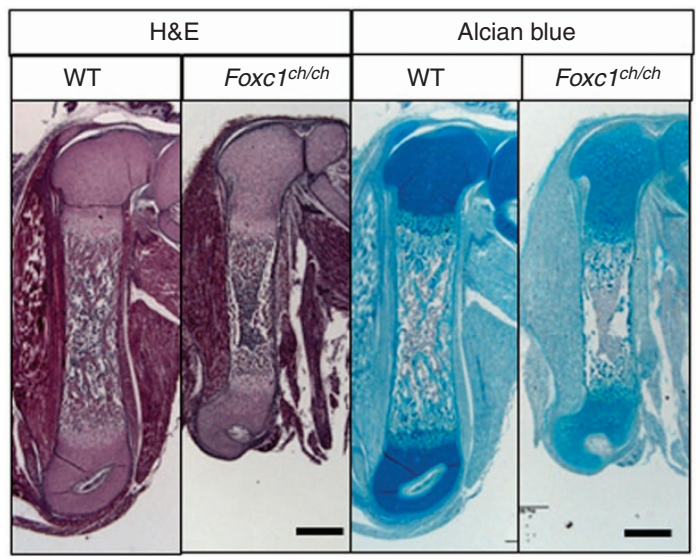

C

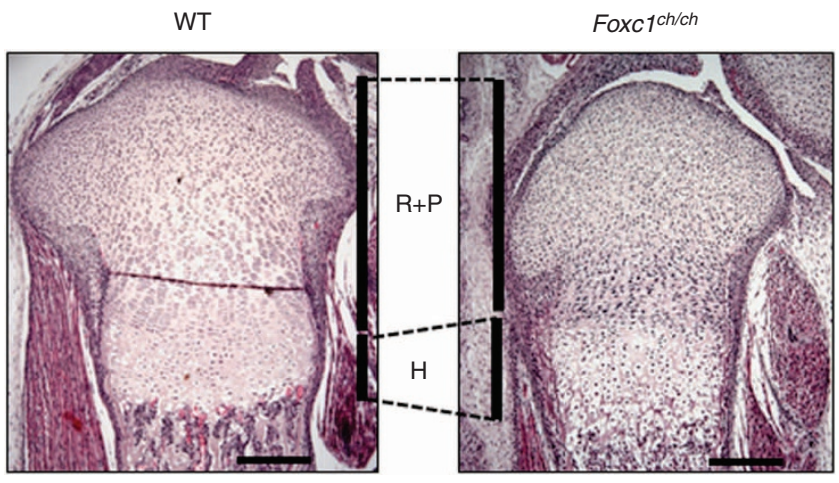

b

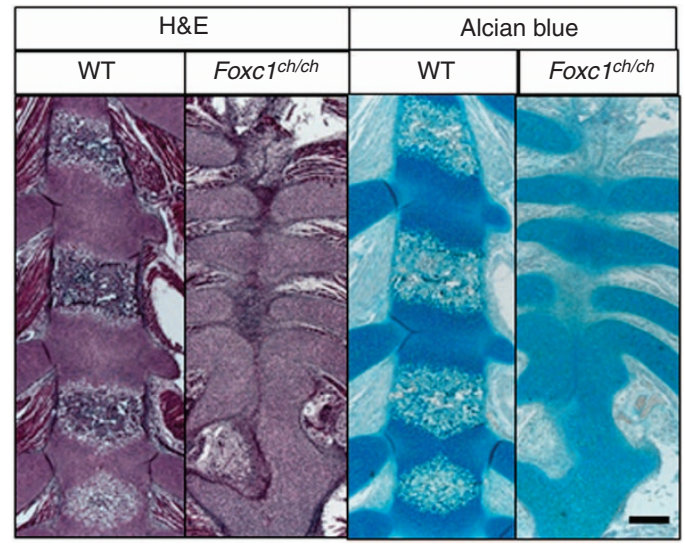

d

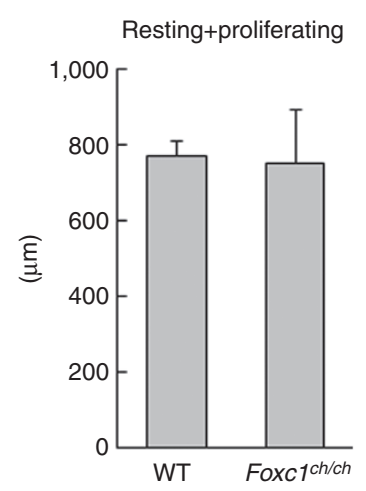

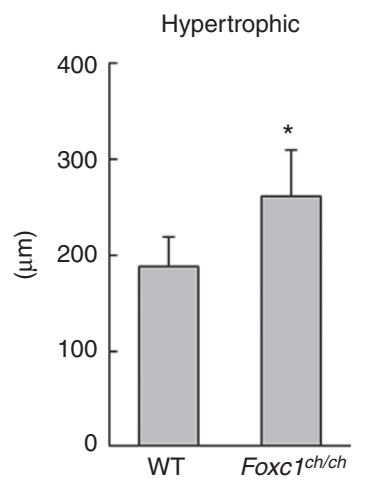

e

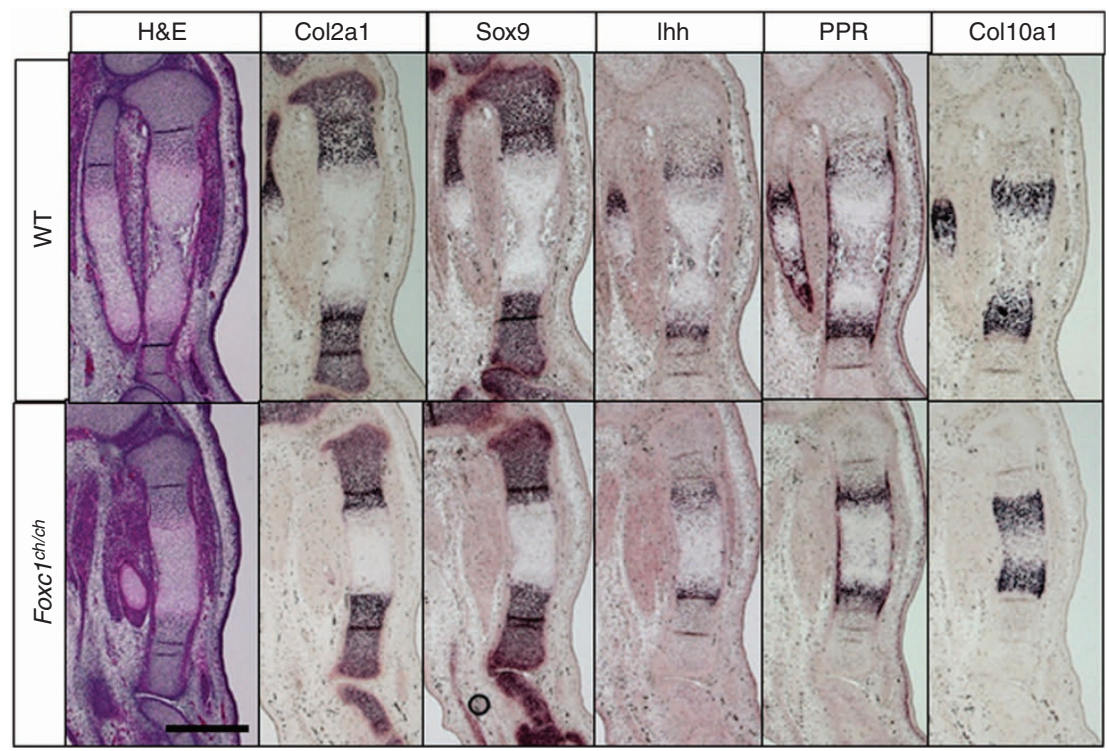

f

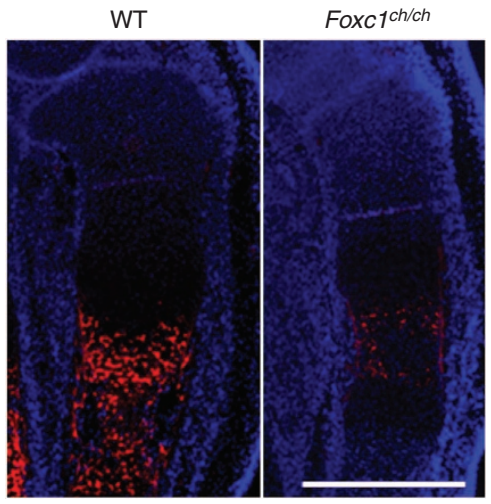

Anti-MMP13

Figure 2 | Delayed endochondral ossification in Foxc1 ${ }^{\text {ch/ch }}$ mice. (a,b) Histological analysis of newborn WT and Foxc $7^{\text {ch/ch }}$ littermate femurs (a) and sternums (b). Paraffin sections of femurs and sternums from newborn WT and Foxc $7^{\text {ch/ch }}$ littermates were examined by haematoxylin and eosin (H\&E) and Alcian blue staining. Scale bar, (a) $500 \mu \mathrm{m}$. (b) $200 \mu \mathrm{m}$. (c) Histological analysis of tibial growth plate chondrocytes of newborn WT and Foxc $7^{\text {ch/ch }}$ littermates. $\mathrm{H}$, hypertrophic chondrocytes; P, proliferating chondrocytes; R, resting chondrocytes. Scale bar $200 \mu \mathrm{m}$. (d) Quantitative analysis of the lengths of the resting and proliferating zone (Resting + Proliferating) and the hypertrophic zone (Hypertrophic) was performed using H\&E-stained sections of the tibia. Data are shown as the mean \pm s.d. $(n=6)$. ${ }^{\star} P<0.05$ (versus WT); Student's $t$-test. (e) Paraffin sections of tibia from E15.5 WT and Foxc $7^{\text {ch/ch }}$ littermate embryos were examined by H\&E staining and in situ hybridization using antisense probes against Col2a1, Sox9, Ihh, PPR and Col10a1. Scale bar, $500 \mu \mathrm{m}$. (f) Paraffin sections of tibia from E15.5 WT and Foxc ${ }^{\text {ch/ch }}$ littermates were subjected to immunofluorescence analyses using an anti-MMP13 antibody. Scale bar, $500 \mu \mathrm{m}$. 
a

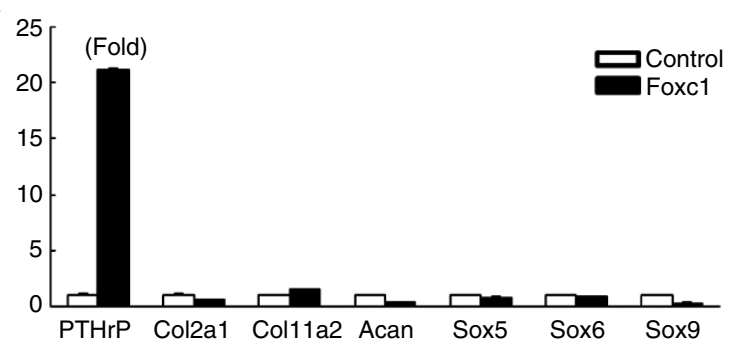

C

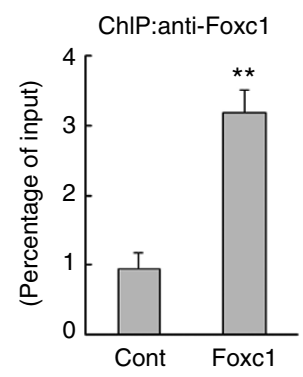

e

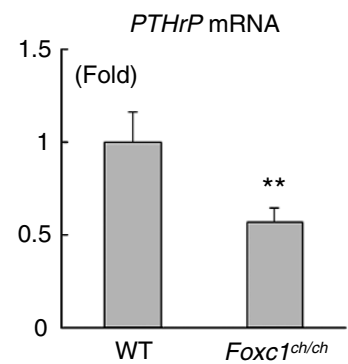

h

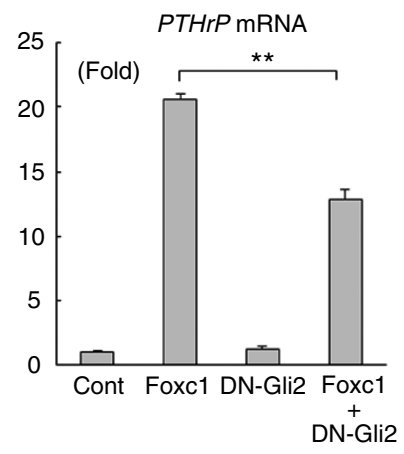

d 6xFoxc1-BE

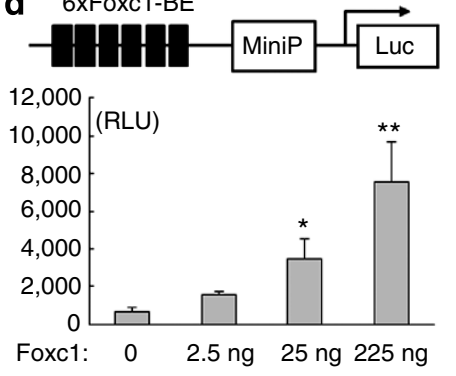

i

6xMyc-Gli2: $\quad-\quad++$ 3xFlag-Foxc1: $\quad++-+$

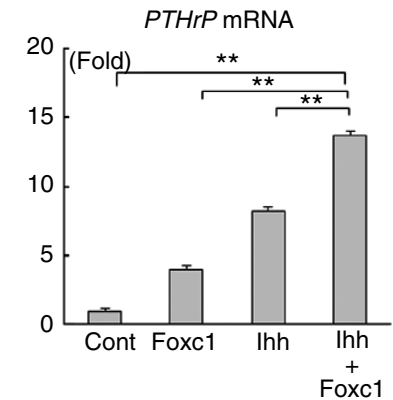

g

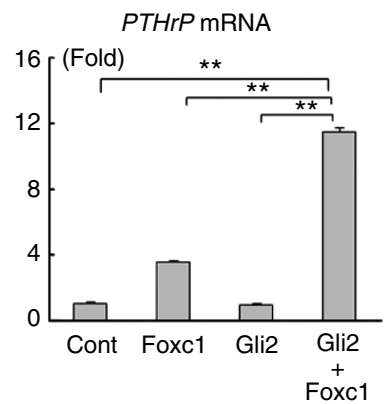
$(\mathrm{KDa})$

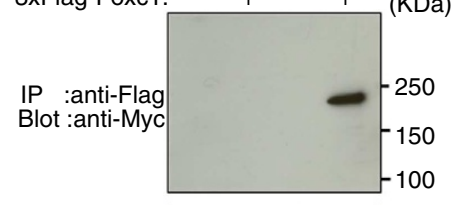

j RFP-Foxc1

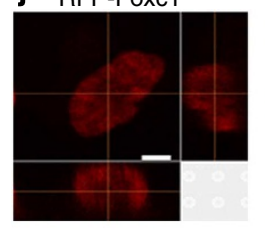

Venus-Gli2

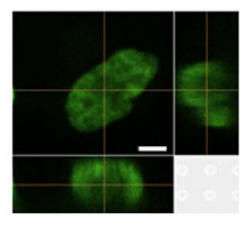

Merge

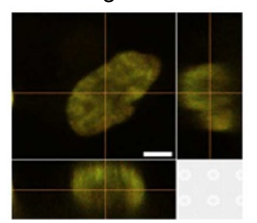

Figure 3 | Foxc1 regulates PTHrP expression through physical and functional interaction with Ihh-Gli2 signalling. (a) Effects of Foxc1 on chondrocyte gene expression in primary chondrocytes analysed by RT-qPCR. (b) DNA pull-down assays using the Foxc1-binding element in the PTHrP gene promoter. Schematic presentation of the putative Foxc1-binding element (CTAAATAAC) in the mouse PTHrP gene promoter (top panel). 293FT cell lysates precipitated with biotin-labelled Foxc1-BE oligonucleotide (middle panel) and cell lysates (bottom panel) were determined by immunoblotting with an antiFlag antibody. (c) Foxc1 was overexpressed in primary chondrocytes and ChIP assays were performed using anti-Foxc1 antibody. The binding of Foxc1 to the PTHrP gene promoter was examined by qPCR. ${ }^{\star \star} P<0.01$ (versus Control); Student's $t$-test. (d) COS-7 cells were transfected with reporter constructs containing 6xFoxc1-BE from the PTHrP gene promoter (upper panel) together with an empty vector and Foxc1. RLU, relative luciferase units. ${ }^{\star} P<0.05$, ${ }^{\star \star} P<0.01$ (versus $0 \mathrm{ng}$ ) (e) PTHrP mRNA expression was determined by RT-qPCR using microdissected hindlimbs isolated from E15.5 Foxc ${ }^{c h} / \mathrm{ch}$ and WT littermate fetuses ${ }^{\star \star} P<0.01$ (versus WT); Student's $t$-test. ( $\left.\mathbf{f}, \mathbf{g}\right)$ Synergistic effect of Foxc1 and Ihh (f) and Foxc1 and Gli2 (g) on PTHrP mRNA expression in primary chondrocytes 3 days after infection with the indicated adenoviruses. ${ }^{\star \star} P<0.01$ (versus Control, Foxc1 or Ihh) ${ }^{\star \star} P<0.01$ (versus Control, Foxc1 or Gli2). (h) Effect of DN-Gli2 on Foxc1-dependent PTHrP mRNA expression in primary chondrocytes 6 days after infection with the indicated adenoviruses; ${ }^{\star \star} P<0.01$ (versus Foxc1). Data for $(\mathbf{a}, \mathbf{c}, \mathbf{d}-\mathbf{h})$ are shown as mean \pm s.d. $(n=3)$ and statistical analysis $(\mathbf{d}, \mathbf{f}, \mathbf{g}, \mathbf{h})$ was performed by one-way analysis of variance followed by the Tukey-Kramer test. (i) 293FT cell lysates were immunoprecipitated with an anti-Flag antibody and then immunoblotted with an anti-Myc antibody (top panel). The cell lysates were immunoblotted with an anti-Myc (middle panel) or anti-Flag (bottom panel) antibody. (j) HEK293 cells were transfected with both RFP-tagged-Foxc1 (RFP-Foxc1) and Venus-tagged Gli2 (Venus-Gli2). Confocal cross sections of $x y$ (left), $y z$ (right) and $x z$ (bottom) were visualized under a confocal microscope. Scale bar, $5 \mu \mathrm{m}$. 
chondrocytes and evaluated the expression of hypertrophic chondrocyte marker genes. Foxc1 markedly upregulated Col10a1 expression (Fig. 5a). The Col10al promoter has a putative
Foxc1-binding motif in the opposite orientation to that of Col10a1 transcription (Fig. 5b). DNA pull-down assays using a biotinylated form of this Foxc1-binding element demonstrated a

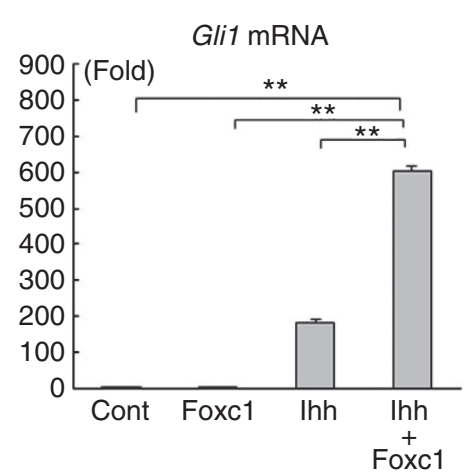

C
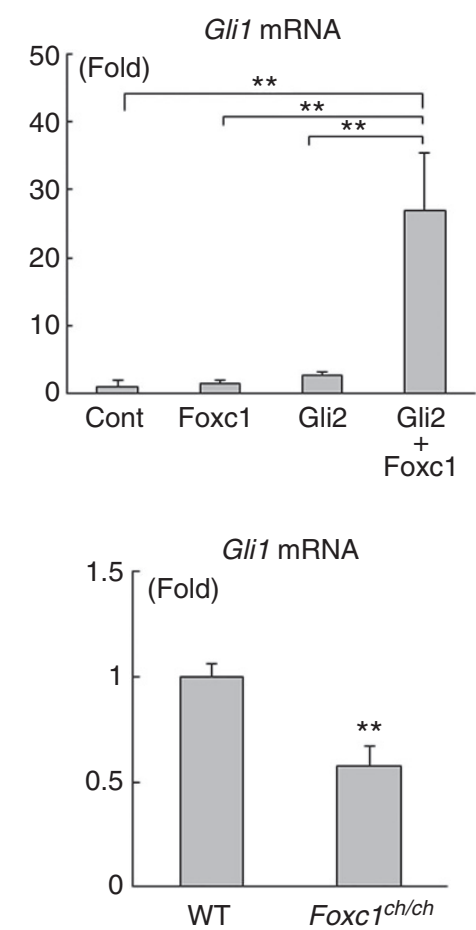

b

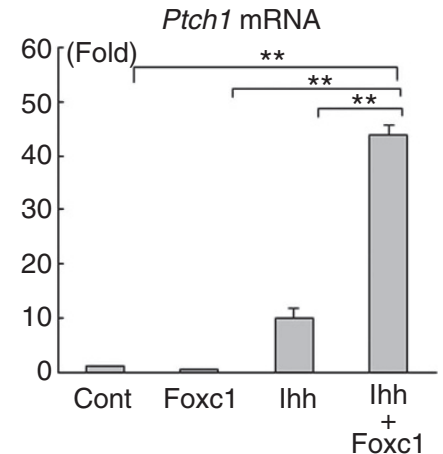

d

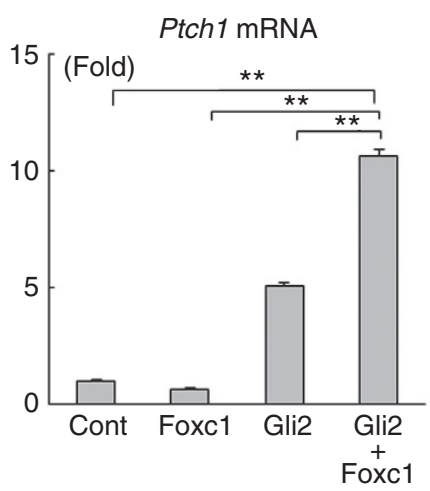

f

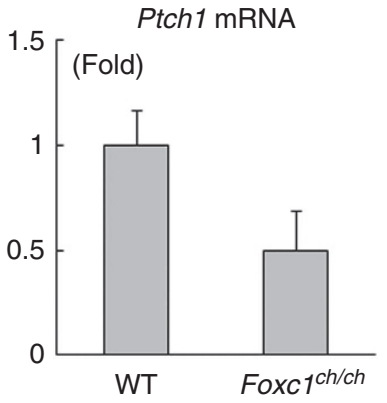

g

g PTHrP promoter ChIP:anti-Gli2

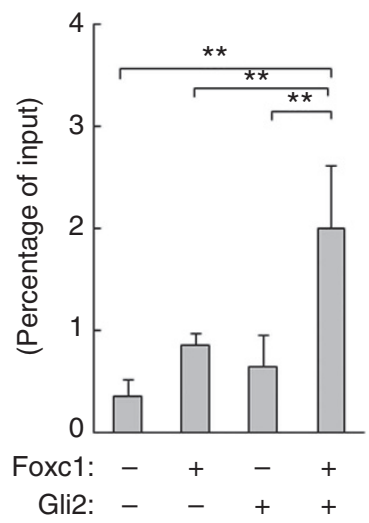

h Gli1 promoter Gli-BE ChIP:anti-Gli2

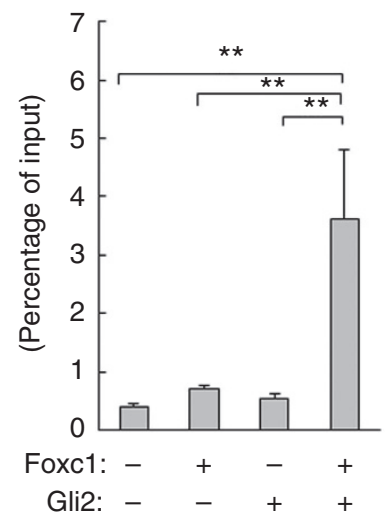

i

Ptch1 promoter Gli-BE ChIP:anti-Gli2

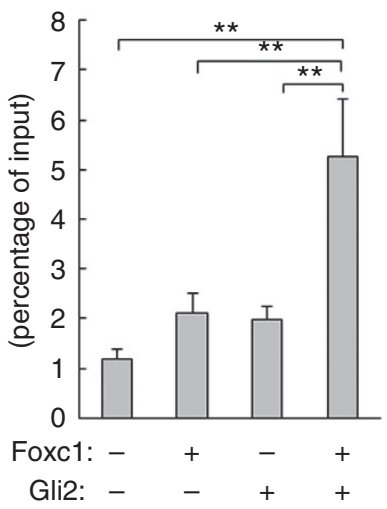


the direct binding of Foxc1, which could be competed with by an excess of non-biotinylated probe (Fig. 5c). ChIP assays using an anti-Foxc1 antibody also revealed that Foxc1 bound to the Col10a1 promoter (Fig. 5d). Moreover, Foxc1 increased the promoter activity of a reporter construct containing six copies of the Col10a1 promoter Foxc1-binding element (Fig. 5e), similar to the experiment in Fig. 3d. Taken together, these results suggest that Colloal is a direct target gene of Foxc1 during the late stage of endochondral ossification.

To determine whether Foxc1 regulates Col10a1 expression during endochondral ossification in vivo, we carried out immunofluorescence analysis of tibial growth plates from newborn mice. This revealed that the type-10 collagen-positive zone was significantly shorter in Foxc1 $1^{\text {ch/ch }}$ compared with WT mice, while the type- 2 collagen-positive zone was unchanged (Fig. 5f,g). In addition, impaired Col10 expression in growth plate chondrocytes was also observed in E15.5 and E17.5 tibia of Foxc1 ${ }^{\text {ch/ch }}$ mice (Supplementary Figs $4 c$ and 8). Analysis of the newborn sternum demonstrated that type-2 collagen-positive chondrocytes failed to undergo sternum development and that type-10 collagen-positive chondrocytes were not detected in Foxc1 ${ }^{\text {ch/ch }}$ mice (Fig. 5h), suggesting impaired chondrocyte hypertrophy. RT-qPCR analysis using primary chondrocytes revealed that Colloal mRNA levels were strikingly decreased in Foxc1 ${ }^{\text {ch/ch }}$ compared with WT mice (Fig. 5i). Importantly, the decrease of Col10al expression in Foxc1 ${ }^{\text {ch }}$ ch mice was restored by the overexpression of Foxc1 (Fig. 5i). Collectively, these results showed that Foxc1-dependent regulation of Col10a 1 expression is important for endochondral ossification.

DN-Foxc1 reduced Ihh target gene expression. To gain further insight into the role of Foxcl in chondrogenesis, we examined the effect of a Foxc1 mutant on Ihh target gene expression. We generated a DN mutant of Foxc1 (DN-Foxc1) containing a DNAbinding domain and a transcriptional inhibitory domain ${ }^{33}$ (Supplementary Fig. 9a,b). DN-Foxc1 inhibited the transcriptional activity of WT Foxcl as assessed by a reporter assay (Supplementary Fig. 9c). Overexpression of DN-Foxc1 decreased PTHrP and Col10al expression induced by Foxcl in primary chondrocytes (Supplementary Fig. 9d,e). Of note, DNFoxcl also decreased the expression of PTHrP, Gli1, Ptch1 and Col10a1 induced by Ihh (Supplementary Fig. $9 \mathrm{f}-\mathrm{i}$ ). These results indicate the importance of Foxcl in the chondrogenic action of Ihh.

Functional redundancy between Foxc1 and Foxc2. Although we have shown that Foxcl controls $\mathrm{PTHrP}$ expression, which is essential for endochondral ossification, the skeletal phenotype of Foxc1 $1^{\text {ch/ch }}$ long bones was relatively mild compared with the severe skeletal defects previously observed in PTHrP-deficient mice ${ }^{34,35}$. Of the forkhead transcription factor superfamily ${ }^{36}$,
Foxc2 is the most closely related to Foxc1 and Nifuji et al reported the expression of Foxc 2 in perichondrium ${ }^{37}$. Thus, we reasoned that the mild skeletal defect observed in Foxc1 ${ }^{\text {ch/ch }}$ mice was likely caused by the functional redundancy between Foxc1 and Foxc2. To support this notion, our microarray analysis and RT-qPCR analysis revealed high levels of Foxc2 expression in chondrogenic cells (Table 1 and Supplementary Fig. 10a). Foxc2 was also relatively highly expressed in the cartilage tissues of newborn mice (Supplementary Fig. 10b), while immunohistochemical analysis using an anti-Foxc2 antibody revealed Foxc2 expression in resting, proliferating and hypertrophic chondrocytes of the tibial growth plate (Supplementary Fig. 10c). Interestingly, Foxc2 increased PTHrP and Colloal expression in primary chondrocytes and stimulated reporter activity through either $\mathrm{PTHrP}$ or Col10a1 promoter Foxc1binding elements (Supplementary Fig. 10d-g). However, the synergistic effects of Foxc2 and Gli2 on Ihh target gene expression, including for PTHrP, Gli1 and Ptch1, were significant but very weak (Supplementary Fig. 10h-j) compared with those of Foxc1 (Figs $3 \mathrm{~g}$ and $4 \mathrm{c}, \mathrm{d}$ ). We next compared the expression level of Foxc1 and Foxc2 in chondrocytes. We found stronger expression of Foxc1 in growth plate chondrocytes compared with that of Foxc2 as determined by in situ hybridization analysis (Supplementary Fig. 11a). RT-PCR analysis also showed a low level of Foxc2 mRNA in rib cartilage and primary chondrocytes, while the level of Foxc2 mRNA was approximately equivalent to that of Foxc1 mRNA in kidney (Supplementary Fig. 11b). Taken together, these data suggest that Foxc2 has a similar function but does not completely overlap with that of Foxc1 in endochondral ossification.

A Foxc1 missense mutation failed to interact with Gli2. The Axenfeld-Rieger syndrome, characterized by glaucoma, hypodontia and skeletal abnormalities, including facial dysmorphism and short stature, is caused by mutations in FOXC1 (refs 27,38,39). Interestingly, mutations of PTHLH, a human homologue of PTHrP, cause brachydactyly and a similar disorder in which patients have a short stature and missing teeth ${ }^{40}$. These clinical observations suggest clinical relevance for the Foxc1-PTHrP axis in the Axenfeld-Rieger syndrome. Thus, we next investigated the pathological role of the FOXC1 mutation, Phe112Ser (F112S), previously identified in the Axenfeld-Rieger syndrome ${ }^{26,41}$. We confirmed that the expression of the mutant protein F112S was equivalent to that of WT Foxc1 (Fig. 6a). Transfection of the F112S mutant decreased $P T H r P$ expression and transcriptional activity of Foxc1, suggesting that F112S acts in a dominant negative manner (Fig. 6b,c). F112S also decreased Ihh-dependent PTHrP expression (Fig. 6d). Moreover, the functional interaction between Foxc1 and Gli2 was strikingly attenuated in the F112S transfectant, which, in turn, impaired PTHrP induction (Fig. 6e).

\footnotetext{
Figure 4 | Foxc1 controls the expression of Ihh target genes by recruiting Gli2. (a,b) Synergistic effect of Foxc1 and Ihh on Gli1 (a) and Ptch1 (b) mRNA expression in primary chondrocytes infected with adenoviruses as indicated. Data are shown as the mean \pm s.d. $(n=3)$. ${ }^{\star *} P<0.01$ (versus Control, Foxc1 or Ihh); one-way analysis of variance (ANOVA) followed by the Tukey-Kramer test. (c,d) Synergistic effect of Foxc1 and Gli2 on Gli1 (c) and Ptch1 (d) mRNA expression in primary chondrocytes infected with adenoviruses as indicated. Data are shown as the mean \pm s.d. $(n=3)$. ${ }^{\star \star} P<0.01$ (versus Control, Foxc1 or Gli2); one-way ANOVA followed by the Tukey-Kramer test. (e,f) Total RNA was isolated from the microdissected hindlimbs of E15.5 WT and Foxc ch $^{2}$ ch littermate embryos, and Gli1 (e) and Ptch1 (f) mRNA expression was determined by RT-qPCR. Data are shown as fold expression normalized to WT (mean \pm s.d., $n=3$ ). ${ }^{\star \star} P<0.01$ (versus WT); Student's $t$-test. (g-i) Primary chondrocytes were infected with control, Foxc1, Gli2 or both Foxc1 and Gli2 adenoviruses and cultured for 3 days. ChIP assays were conducted using an anti-Gli2 antibody and DNA binding to gene promoters was determined by qPCR using primer pairs specific for $\operatorname{PTHrP}(\mathbf{g}), \mathrm{Gli1}(\mathbf{h})$ and Ptch1 (i) gene promoters, which contain the Gli-binding element. Data are shown as the mean \pm s.d. $(n=3)$. ${ }^{\star \star} P<0.01$ (versus control, Foxc1, or Gli2); one-way ANOVA followed by the Tukey-Kramer test.
} 
a

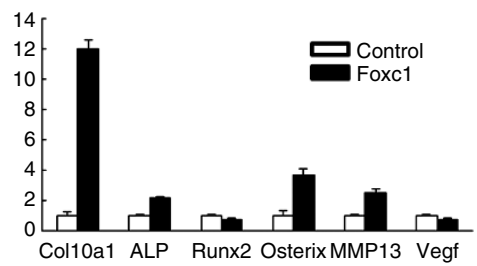

C

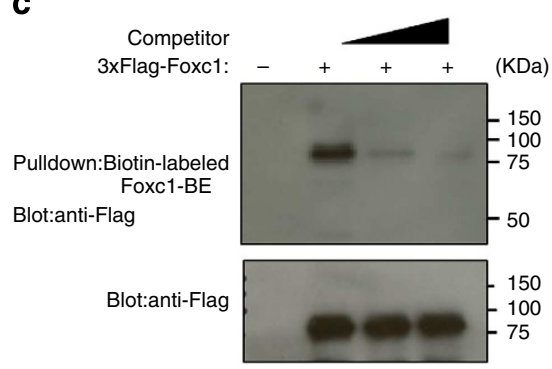

$\mathbf{f}$

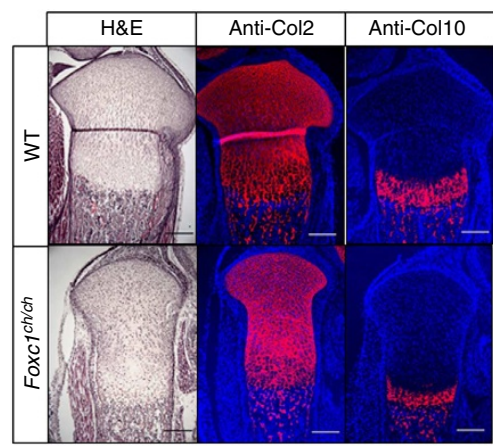

h

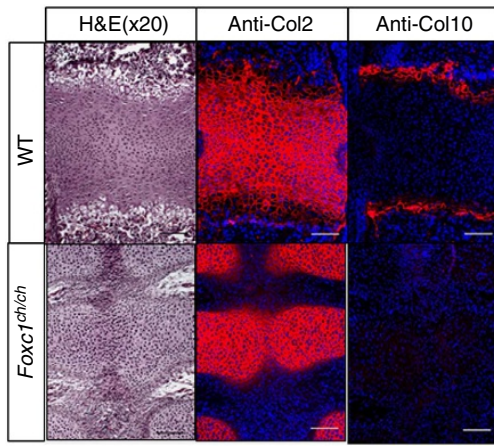

b

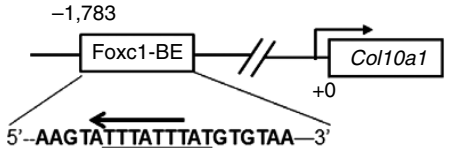

d

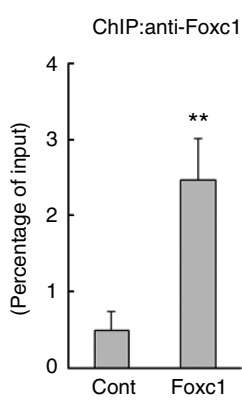

e
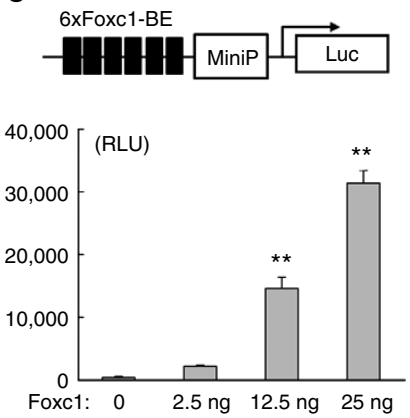

$\mathbf{9}$

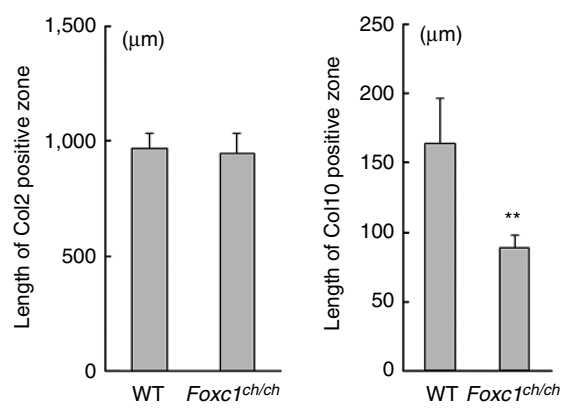

i

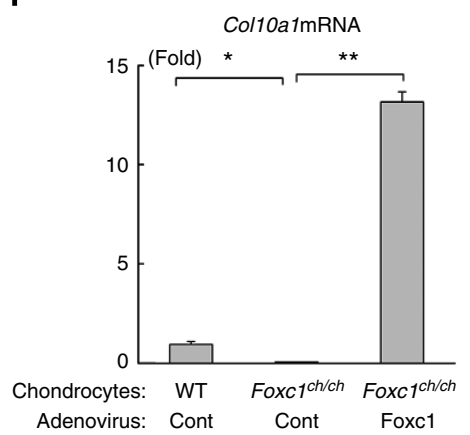

Figure 5 | Col10a1 is the direct target of Foxc1 in vitro and in vivo. (a) Effects of Foxc1 on hypertrophic chondrocyte gene expression. Primary chondrocytes were infected with control or Foxc1 adenovirus and chondrocyte gene expression was analysed by RT-qPCR. Data are shown as the mean \pm s.d. $(n=3)$. (b) Schematic presentation of the putative Foxc1-binding element (ATTTATTTA) in the promoter region of mouse Col10a1. (c) DNA pull-down assays using the Foxc1-binding element in the Col10a1 promoter. 293FT cell lysates precipitated with biotin-labelled Foxc1-BE oligonucleotide (upper) and total cell lysates (lower) were assessed by immunoblotting with an anti-Flag antibody. (d) ChIP assays using anti-Foxc1 antibody. The binding of Foxc1 to the Col10a1 promoter was examined by qPCR. Data are shown as the mean \pm s.d. $(n=6) .{ }^{\star \star} P<0.01$ (versus Control); Student's $t$-test. (e) COS-7 cells were transfected with reporter constructs containing 6xFoxc1-BE from the Col10a1 gene promoter (upper panel) together with an empty vector and Foxc1. Data are expressed in relative luciferase units (mean \pm s.d., $n=3$ ). ${ }^{\star \star} P<0.01$ (versus $0 \mathrm{ng}$ ); one-way analysis of variance (ANOVA) followed by the Tukey-Kramer test. (f) Immunofluorescence analysis of tibial growth plate chondrocytes in newborn WT and Foxc $7^{\text {ch/ch }}$ littermates. Paraffin sections of tibia were subjected to haematoxylin and eosin (H\&E) staining and immunofluorescence analyses using anti-Col2 and anti-Col10 antibodies. Scale bar, $200 \mu \mathrm{m}$. (g) Quantitative analysis of the lengths of Col2-positive and Col10-positive zones. Data are shown as the mean \pm s.d. $(n=5){ }^{\star \star} P<0.01$ (versus WT); Student's t-test. (h) Immunofluorescence analysis of sternums in newborn WT and Foxc $7^{\text {ch/ch }}$ mice. Paraffin sections of sternums of the mice were subjected to immunofluorescence analyses using an anti-Col2 or anti-Col10 antibody. The H\&E staining images are higher magnification images of those

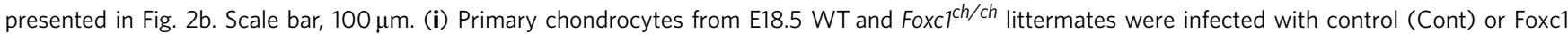
adenovirus as indicated. Col10a1 mRNA expression was determined by RT-qPCR. Data are shown as the mean \pm s.d. $(n=3){ }^{\star \star} P<0.01$, ${ }^{\star} P<0.05$; one-way ANOVA followed by the Tukey-Kramer test. 
a

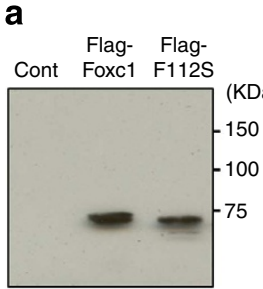

Blot: anti-Flag

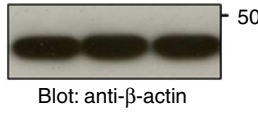

b

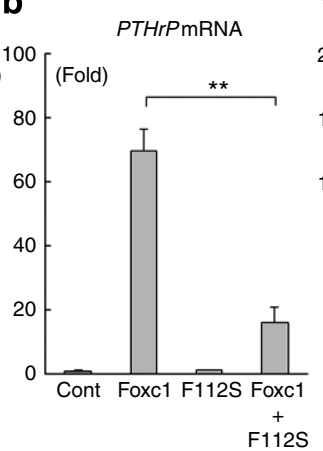

C

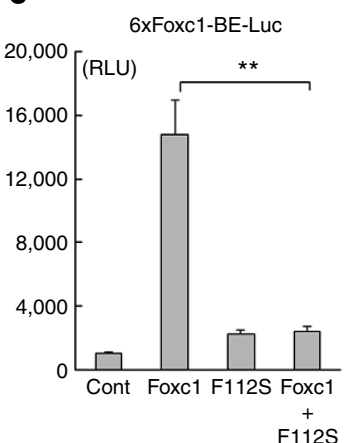

d

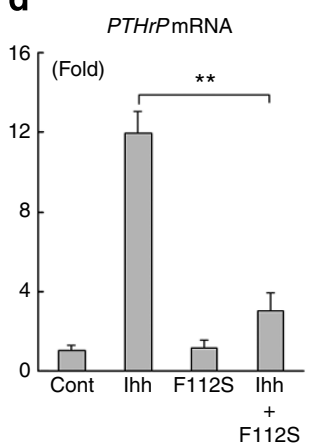

e

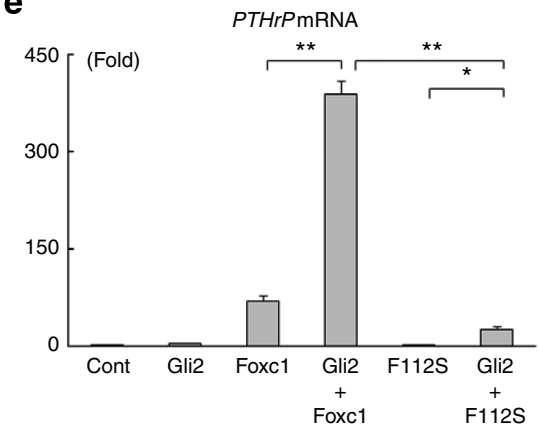

f

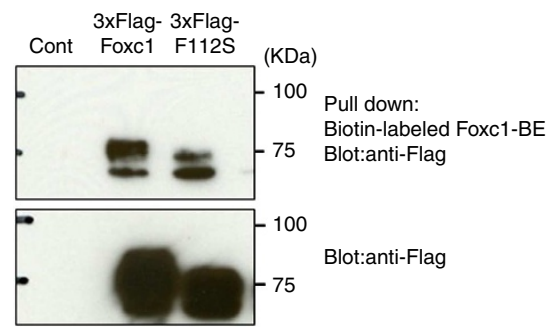

g
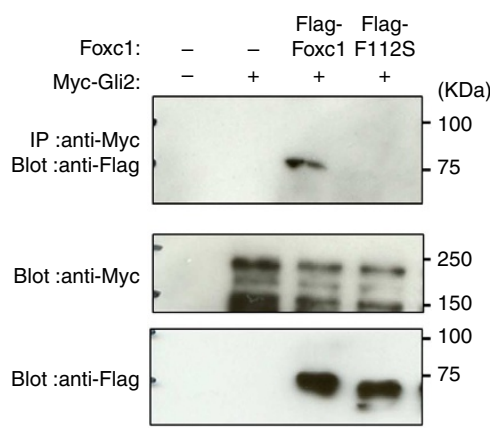

h

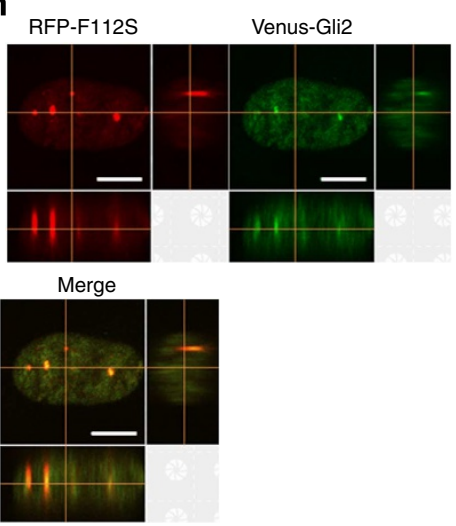

Figure 6 | Impaired functional interaction between a pathological missense Foxc1 mutant and Gli2. (a) 293FT cells were transfected with empty vector, Flag-tagged WT Foxc1 (Flag-Foxc1) or Flag-tagged-F112S mutant Foxc1 (Flag-F112S), and cell lysates were immunoblotted with anti-Flag (upper panel) and anti- $\beta$-actin (lower panel) antibodies. (b) Effect of F112S mutant on PTHrP. PTHrP mRNA expression was determined by RT-qPCR. Data are shown as fold activation normalized to control (mean \pm s.d., $n=3$ ). ${ }^{\star \star} P<0.01$ (versus Foxc1); one-way analysis of variance (ANOVA) followed by the Tukey-Kramer test. (c) Effect of the F112S mutation on Foxc1 transcriptional activity on the PTHrP promoter. Data are expressed in relative luciferase units (mean \pm s.d., $n=3$ ). ${ }^{\star \star} P<0.01$ (versus Foxc1); one-way ANOVA followed by the Tukey-Kramer test. (d) Inhibitory effect of F112S on PTHrP mRNA expression induced by Ihh. Data are shown as the mean \pm s.d. $(n=3) .{ }^{\star} P<0.01$ (versus Ihh); one-way ANOVA followed by the Tukey-Kramer test. (e) Impaired synergistic effect between F112S and Gli2 on PTHrP mRNA expression. Primary chondrocytes were infected with the indicated adenoviruses and PTHrP mRNA expression was analysed by RT-qPCR analysis. Data are shown as the mean \pm s.d. $(n=3) .{ }^{\star \star} P<0.01,{ }^{\star} P<0.05$; one-way ANOVA followed by the Tukey-Kramer test. (f) DNA pull-down assays using the Foxc1-binding element from the PTHrP promoter. Samples precipitated with the biotin-labelled Foxc1-BE oligonucleotide (upper panel) and total cell lysates (lower panel) were immunoblotted with an anti-Flag antibody. (g) F112S failed to interact with Gli2. Lysates of the 293FT cells transfected as indicated were immunoprecipitated with an anti-Myc antibody and immunoblotted with an anti-Flag antibody (top). The cell lysates were immunoblotted with an anti-Myc (middle) or anti-Flag (bottom) antibody. (h) Nuclear localization of F112S and Gli2. HEK293 cells transfected with RFP-tagged F112S (RFP-F112S) and Venus-tagged Gli2 (Venus-Gli2). Confocal cross sections of $x y$ (top left), yz (right) and $x z$ (bottom) were visualized under a confocal microscope. Scale bar, $5 \mu \mathrm{m}$.

Although F112S could bind the Foxc1-binding element in the PTHrP promoter (Fig. 6f), IP-western analysis demonstrated that F112S failed to physically interact with Gli2 (Fig. 6g). RFPtagged-Foxc1-F112S localized to the nucleus but showed an abnormal aggregation pattern, which resulted in a marked reduction of nuclear co-localization with Venus-tagged Gli2
(Fig. 6h). Furthermore, F112S failed to recruit endogenous Gli2 to the Gli-binding element located in the PTHrP, Gli1 and Ptch1 gene promoters (Supplementary Fig. 12). These results collectively suggest that the loss of functional interaction between F112S and Gli2 contribute, at least in part, to the pathogenesis of the Axenfeld-Rieger syndrome. 


\section{Discussion}

Endochondral ossification is characterized by sequential chondrocyte differentiation including resting, proliferating and hypertrophic processes. Chondrocytes form growth plate layers in which specific marker gene expression is strictly controlled by the communication of various transcription factors. Many details of this transcriptional network remain to be elucidated and to achieve a full understanding of the molecular basis of endochondral ossification it is important to identify and characterize the full repertoire of transcription factors involved.

In this study, we performed FACS-assisted microarray profiling using transgenic mouse embryos that selectively express the Venus gene in developing chondrocytes. We identified Foxcl as a functional transcription factor selectively expressed in chondrocytes under physiological conditions. Foxc1 has a diverse range of biological functions and is essential for the development of several tissues including the heart, blood vessels ${ }^{42}$, kidney and urinary tract $^{43}$, the eye ${ }^{44}$ and bones ${ }^{45}$. The mechanism by which Foxc1 regulates skeletal development has previously been investigated from the viewpoint of intramembranous bone formation with particular focus on osteoblasts, not endochondral ossification. Thus, several groups have reported the importance of bone morphogenetic protein signalling and Msx2 in Foxc1-dependent calvarial bone development ${ }^{46-48}$. Although Alcian blue-positive nodule formation of mesenchymal cells isolated from Foxcl lacZ embryos has been studied ${ }^{45}$, the molecular mechanisms by which Foxc1 controls endochondral formation are largely unknown. Our results demonstrated that Foxc1 functionally associates with the Ihh-Gli2 signalling pathway during chondrocyte development. Because Ihh has been reported to regulate not only early chondrogenesis through the induction of PTHrP but also chondrocyte hypertrophy in a PTHrP-independent fashion ${ }^{11,14}$, our findings of Foxc1-dependent regulation of both PTHrP and Col10a1 are consistent with the multiple functions of Ihh in endochondral ossification. Gli2 is the major signalling molecule that transduces the biological function of the hedgehog family of proteins. Interestingly, similar skeletal abnormalities, including delayed endochondral ossification, defects in the vertebral column, and a reduced tibial length, were also observed in Gli2-deficient mice ${ }^{17,49}$. Importantly, we found that the loss of this functional interaction caused by a missense mutation of Foxcl is likely to contribute to the pathogenesis of the Axenfeld-Rieger syndrome. In addition, a DN-Foxc1 diminished chondrogenic action of Ihh. Taken together, our study indicates a novel paradigm; interaction between Foxc1 and Ihh-Gli2 signalling is required for normal skeletal development (Supplementary Fig. 13).

Our screening system did not include a cell expansion step, which has several advantages over gene expression profiling using cultured cells. Cartilage tissues exist in unique physiological conditions that cannot be mimicked in vitro. These include a three-dimensional structure, abundant extracellular matrix, the absence of vessels and hypoxia. However, because it is generally accepted that these conditions affect chondrocyte gene expression, our screening system enables us to identify transcription factors that genuinely function in vivo. Indeed, Venus-positive cells showed high expression of Sox5, Sox6 and Sox9, which are known essential transcription factors involved in chondrogenesis (Table 1). Moreover, several reports have indicated that the combination of tissue-specific reporter mice and FACS-assisted microarray profiling is a useful tool in gene screening. For example, Nakamura et al. ${ }^{50}$ identified $W w p 2$ as a Sox 9 target gene in chondrocytes using Sox9-3' EGFP knock-in mice, while Ieda et al. ${ }^{51}$ identified three transcription factors that enable the direct reprogramming of dermal fibroblasts into functional cardiomyocytes using the $\alpha M H C$ promoter. Although we focused on Foxc1 in this study, other genes identified by microarray analysis might play important roles in endochondral ossification.

Although we showed that Foxc1 regulates Gli2 function by promoting DNA binding of Gli2 to target gene promoters, details of the mode of action remain to be elucidated. One possibility is that Foxc1 promotes chromatin accessibility as a pioneer factor and thereby regulates gene expression. Pioneer factors are known to open up local chromatin structure through chromatin remodelling, and many forkhead family transcription factors function as pioneer factors ${ }^{52}$. For example, FOXA1 is required for the optimal chromatin conditions needed for the DNA binding and gene expression induced by nuclear receptors, including oestrogen and androgen receptors ${ }^{53,54}$. Because Foxc1 does not contain a functional domain that regulates chromatin remodelling, it might recruit a variety of chromatin-modifying enzymes to regulate gene expression. It would be interesting to further investigate the transcriptional partner(s) of Foxcl in chondrocytes during endochondral ossification.

Our present results indicate that Foxc1 directly regulates PTHrP expression in chondrocytes (Fig. 3). PTHrP is expressed in perichondrial cells and delays chondrocyte hypertrophy by maintaining their proliferation. Our detection of Foxc1 in perichondrial cells (Supplementary Fig. 2a) and in the elongated hypertrophic chondrocyte zone in the tibial growth plate of Foxc1 ${ }^{\text {ch/ch }}$ mice (Fig. 2d) support the important role of the Foxc1PTHrP axis during endochondral ossification. However, the skeletal phenotype of the sternum in Foxc1 ${ }^{\text {ch/ch }}$ mice showing a complete absence of hypertrophic chondrocytes, is not consistent with PTHrP inhibiting chondrocyte hypertrophy. One explanation for this is that morphogenetic processes that are different between the sternum and long bones influence the phenotype differences. For example, during sternal development, bilateral sternal cartilage bars migrate towards the midline and fuse to form the sternum; this is followed by chondrocyte hypertrophy and ossification ${ }^{55}$, suggesting a critical role for cell migration during the development of the sternum. Interestingly, Foxcl is expressed in the sternum primordium of the E12.5 mouse embryo ${ }^{45}$ and several reports indicate that it is involved in the migration of various types of cells, including germ $^{56}$, endothelial ${ }^{57}$ and breast cancer cells ${ }^{58}$. These data suggest that Foxc1-dependent cell migration also contributes to skeletal development. Reduced cell migration activity could, therefore, partly account for the failure of sternum development in Foxc1 ${ }^{\mathrm{ch} /}$ ch mice (Fig. 2b). We found that Foxc1 is widely expressed in growth plates; therefore, it is likely that Foxc1 has multifunctional roles in several stages of cartilage development.

Foxc1 ${ }^{\text {ch/ch }}$ mice showed elongation of the hypertrophic chondrocyte zone (Fig. 2c,d), whereas the Col10-positive chondrocyte area was significantly decreased in E15.5 (Supplementary Fig. 4c), E17.5 (Supplementary Fig. 8) and P0 Foxc $1^{\text {ch/ch }}$ tibia (Fig. 5f,g). There is a discrepancy between these phenotypes because type-10 collagen is a well-established specific marker for hypertrophic chondrocytes. Mechanistically, we speculate that reduced Col10 expression makes hypertrophic chondrocytes functionally immature, and subsequently causes their terminal differentiation to be delayed. To support this notion, MMP13 expression was markedly decreased in Foxc $1^{\text {ch/ch }}$ mice (Fig. 2f). The extended hypertrophic chondrocyte zone is likely due to impaired terminal differentiation, which causes inhibition of vascular invasion and subsequent replacement with bone. Further analysis is needed to characterize the hypertrophic chondrocytes observed in Foxc1 $1^{\text {ch/ch }}$ mice.

Among the forkhead protein family members, Foxc2 is most closely related to Foxcl, suggesting that the two proteins may functionally overlap. In this study, we found that Foxc2 is expressed in growth plate chondrocytes and increases PTHrP and 
Col10a1 expression in primary chondrocytes (Supplementary Fig. 10). Foxc2-deficient mice also showed skeletal abnormalities $^{59}$ and the deletion of both Foxc1 and Foxc2 in mice resulted in early embryonic lethality ${ }^{43}$. However, the observed skeletal phenotype of $F o x c 1^{\text {ch/ch }}$ did not perfectly match that of Foxc2-deficient mice. For instance, no cleft palate was seen in Foxc1 ${ }^{\text {ch/ch }}$ mice yet this was observed in Foxc2-deficient mice together with normal sternum development ${ }^{59}$. Thus, Foxc1 and Foxc2 have redundant and diverse roles in cartilage development.

We also showed that the F112S mutation in the forkhead DNA-binding domain, responsible for the Axenfeld-Rieger Syndrome, causes loss of the functional association between Foxc1 and Gli2 (Fig. 6). The F112S mutation leads to abnormal craniofacial development and dental abnormalities ${ }^{41}$; however, the molecular basis of this is unknown. It has been reported that disease-causing missense mutations in the forkhead DNAbinding domain of FOXC1 cause various effects on the structure and function of Foxc1 (refs 29,60,61). For example, I91S and $\mathrm{R} 127 \mathrm{H}$ mutations result in nuclear localization defects, while S82T, S131L and R127H mutations reduce the DNAbinding activity. Because the F112S mutation does not affect DNA binding (Fig. 6f), its clinical consequences are likely to result from the failure of transcriptional machinery complex formation, including the involvement of Gli2. It is possible that F112S disrupts the local protein structure, which, in turn, causes abnormal organization of the functional domain located outside the forkhead domain. Moreover, the electrostatic charge distribution on the protein surface might be altered, affecting protein-protein interactions. Molecular modelling or protein structure analysis of the F112S mutation is necessary to uncover the molecular basis of its effect.

In addition to skeletal abnormalities, human FOXC1 mutations lead to dental anomalies including missing or small teeth. Interestingly, human PTHLH mutations are also reported to cause missing teeth ${ }^{40}$. PTHrP and the PTH/PTHrP receptor are expressed in tooth buds ${ }^{62,63}$, and overexpression of a constitutively active $\mathrm{PTH} / \mathrm{PTHrP}$ receptor in mice causes a reduction in the number of ameloblasts and disorganization of the odontoblast layer ${ }^{64}$. These data collectively suggest that the Foxc1-PTHrP axis is also involved in tooth development, and it will be of interest to elucidate the role of Foxcl during tooth morphogenesis to help determine the pathogenesis of the dental anomalies observed in the Axenfeld-Rieger syndrome.

In conclusion, we identified a novel function for Foxcl as a critical transcription factor for endochondral ossification. This function modulates the expression of chondrogenic genes through its physical and functional interaction with Ihh-Gli2 signalling. Our findings contribute to an improved understanding of the molecular mechanisms underlying endochondral ossification and provide new insights into the transcriptional network system of skeletal development.

\footnotetext{
Methods

Generation of Col2a1-venus transgenic mice. Venus cDNA (kindly provided by Dr Atsusi Miyawaki, Lab for Cell Function Dynamics, BSI, RIKEN, Japan) was amplified by PCR and fused to the Col2al promoter. This transgene vector, containing a polyA site, was linearized using NotI digestion and used to generate transgenic mice by pronuclear injection. DNA was injected into single-cell BDF1 zygotes to generate transgenic mice. To determine mouse genotypes, genomic DNA isolated from tail biopsies was analysed by PCR using specific primers for Venus cDNA (sense primer: $5^{\prime}$-TGGTGGTGCAAATCAAAGAA- $3^{\prime}$, antisense primer: $5^{\prime}$-TAGGCTTTCCACGTCGATCT- $\left.3^{\prime}\right)$. All experiments were performed under protocols approved by the Osaka University Graduate School of Dentistry
} animal committee.

Microarray analysis. We dissected rib cartilage, vertebrae and limb buds from E13.5 Col2a1-Venus-Tg mice and digested the tissues into single cells using $0.1 \%$ collagenase and $0.1 \%$ trypsin. Venus-positive and Venus-negative cells were sorted with a FACS Aria flow cytometer (Becton-Dickinson, NY, USA) using the manufacturer's instructions. Total RNA was extracted using NucleoSpin RNA II (Macherey-Nagle, Duren, Germany) without a cell expansion step. Microarray analysis was performed using the Affymetrix Mouse Genome 430 2.0 Array according to the manufacturer's protocol (Affymetrix, Santa Clara, CA, USA). Microarray data have been deposited in NCBI Gene Expression Omnibus (http:// www.ncbi.nlm.nih.gov/geo/) under the accession number GSE65350.

RT-qPCR. Total RNA was denatured at $65^{\circ} \mathrm{C}$ for $5 \mathrm{~min}$ and then cDNA was synthesized using a ReverTra Ace qPCR RT Master Mix (TOYOBO, Osaka, Japan). For quantification of mRNA, RT-qPCR amplification was performed using the TaqMan PCR protocol and the ABI StepOne Plus real-time PCR system (Applied Biosystems, Foster city, CA, USA). Primers and TaqMan probes used for amplification are listed in Supplementary Table 2. mRNA expression was normalized to that of $\beta$-actin. Data are represented as means \pm s.d. $(n=3)$.

Whole-mount in situ hybridization. A 594-bp fragment of Foxc1 was amplified using specific primers (sense primer: $5^{\prime}$-CAGAGCTCCCTCTACAGCTCC- $3^{\prime}$, antisense primer: $5^{\prime}$-TCAGAATTTGCTACAGTCATA- $3^{\prime}$ ) and subcloned into a pCR2 TOPO vector (Invitrogen, CA, USA). A 405-bp Col2a1 fragment subcloned into pBluescript SK vector was kindly provided by Dr Toshihisa Komori (Nagasaki University Dental School, Nagasaki, Japan). Plasmid vectors containing Foxc1 and Col2a1 fragments were linearized using BamHI (Foxc1) and EcoRI (Col2a1), respectively. Digoxigenin (DIG)-labelled antisense RNA probes for Foxc1 or Col2al were generated with SP6 or T7 RNA polymerase, respectively, and using a DIG RNA Labeling kit (Roche, Basel, Switzerland) according to the manufacturer's instructions. C57BL/6 mouse embryos (E12.5) were fixed in $4 \%$ paraformaldehyde (PFA) in PBS overnight at $4{ }^{\circ} \mathrm{C}$. Samples were hybridized overnight at $70{ }^{\circ} \mathrm{C}$ with genespecific DIG-labelled RNA probes, then washed and incubated with anti-DIG antibody conjugated to alkaline phosphatase (anti-DIG-AP antibody, Roche) at a 1:2,500 (v/v) dilution. Nitroblue tetrazolium and 5-bromo-4-chloro- $3^{\prime}$ indolyphosphate $\mathrm{p}$-toluidine were used for signal detection.

In situ hybridization. Tibias from E14.5 or E15.5 mice were fixed in 4\% PFA-PBS overnight at $4{ }^{\circ} \mathrm{C}$, embedded in paraffin and cut into 5 - $\mu \mathrm{m}$ sections. DIG-labelled single-stranded RNA probes were prepared using a DIG RNA labelling kit (Roche) according to the manufacturer's instructions. The sections were deparaffinized and hybridized with chondrocyte gene-specific DIG-labelled RNA probes for $8 \mathrm{~h}$ at $70^{\circ} \mathrm{C}$. The signals were detected with alkaline phosphatase-conjugated anti-DIGAP antibody at a 1:2,500 (v/v) dilution. Plasmid vectors containing fragments of Foxc1 (594 bp), Foxc2 (458 bp), Col2a1 (405bp), Sox9 (456bp), Ihh (579 bp), Ppr (779 bp), Col10a1 (637 bp), Col1a1 (320 bp) and Runx2 (634 bp) were linearized using XbaI (Ppr), EcoRI (Col2a1, Ihh and Col1a1), BamHI (Foxc1, Foxc2, Col10a1 and Runx2) or NcoI (Sox9), respectively. DIG-labelled antisense RNA probes were generated with SP6 (Ppr and Sox9), T3 (Col2a1, Col1a1 and Runx2) or T7 (Foxc1, Foxc2, Ihh and Col10a1) RNA polymerase using a DIG RNA Labeling kit according to the manufacturer's instructions. These chondrocyte gene probes were kindly provided by Dr Noriyuki Tsumaki (Kyoto University, Kyoto, Japan) and Toshihisa Komori (Nagasaki University Dental School, Nagasaki, Japan).

Immunohistochemical and immunofluorescence analyses. Samples were fixed with $4 \%$ PFA-PBS, embedded in paraffin and cut into $4-\mu$ m-thick sections. The sections were deparaffinized and stained with Mayer's haematoxylin and eosin. Immunohistochemistry was performed using the following antibodies: anti-Col2 (\#7050; Chondrex, WA, USA) at a 1:500 (v/v) dilution, anti-MMP13 (\#ab39012; Abcam, Cambridge, UK) at a 1:50 (v/v) dilution and anti-Foxc1 (\#ab5079) at a 1:50 $(\mathrm{v} / \mathrm{v})$ dilution. Antigen retrieval was performed by heat mediation in a citrate buffer ( $\mathrm{pH}$ 6) for Foxcl and 5\% hyaluronidase in PBS for $30 \mathrm{~min}$ at $37^{\circ} \mathrm{C}$ for $\mathrm{Col} 2$ and MMP13. Immunoreactivity was visualized with a biotinylated anti-rabbit IgG secondary antibody using the ABC Vectastain kit (Vector Laboratories, CA, USA) and the peroxidase substrate DAB kit (Vector Laboratories), according to the manufacturer's protocols. For immunofluorescence staining, Alexa Fluor 555conjugated anti-rabbit IgG was used at a 1:500 (v/v) dilution as secondary antibody to visualize immunoreactivity. Counterstaining was performed using $4^{\prime}, 6$-diamidino-2-phenylindole.

Foxc1 $\mathbf{1}^{\text {ch/ch }}$ mice. Foxc1 $1^{\text {ch }}$ (congenital hydrocephalus, ch) heterozygous mice were purchased from the Jackson Laboratory (Bar Harbour, ME, USA) and maintained on a standard 12:12-h light/dark cycle. Homozygous Foxc1 ${ }^{\text {ch/ch }}$ mice were obtained by mating heterozygous Foxc1 $1^{c h /+}$ mice ${ }^{65}$. To determine mouse genotypes, genomic DNA was amplified using a Foxc1-specific primer pair (sense primer: 5'-TATGAGCGTGTACTCGCACCCT-3'; antisense primer: $5^{\prime}$-CGTACCGTTCT CCGTCTTGATGTC- $3^{\prime}$ ) and PCR products were then digested with Cac8I (ref. 43). The WT gene yields $178 \mathrm{bp} 197 \mathrm{bp}$ fragments and the Foxc1 ${ }^{\text {ch }}$ gene yields a 375 bp fragment. All experiments were performed with E15.5, E17.5 and newborn littermates under protocols approved by the Osaka University Graduate School of Dentistry animal committee. 
Skeletal preparation. The skin of newborn and E15.5 mice was removed and fixed with $95 \%$ ethanol overnight. Cartilage tissues were stained with $1.5 \%$ Alcian blue followed by staining of bone tissues with $0.02 \%$ alizarin red. Skeletal preparation samples were photographed under a stereoscopic microscope.

Cell culture and reagents. HEK293, 293FT and COS-7 cells were purchased from the RIKEN Cell Bank (Tsukuba, Japan) or Life Technologies, and cultured in Dulbecco's modified Eagle's medium (Sigma-Aldrich, MO, USA) containing 10\% fetal bovine serum at $37^{\circ} \mathrm{C}$ in a humidified $5 \% \mathrm{CO}_{2}$ incubator.

Isolation of primary chondrocytes. Mouse primary chondrocytes were isolated according to the protocol described by Gartland et al. ${ }^{66}$ In brief, the rib cartilage was dissected from newborn mice and digested with $0.1 \%$ collagenase D (Roche) and $0.5 \%$ trypsin (Life Technologies, CA, USA) for $6 \mathrm{~h}$ at $37^{\circ} \mathrm{C}$ and then centrifuged for $5 \mathrm{~min}$ at $1,500 \mathrm{~g}$. The supernatant was removed and the pellet resuspended with $\alpha$-minimum essential medium containing $10 \%$ fetal bovine serum and antibiotics. Cells within two passages were used as primary chondrocytes for experiments.

Construction of expression vectors and transfection. WT and mutant Foxc1 cDNAs were amplified by PCR and subcloned into the EcoRI and XbaI sites of an expression vector containing $6 \times$ Myc, Flag and $3 \times$ Flag epitopes. The primer pairs used for PCR were as follows (WT Foxc1: sense primer: $5^{\prime}$-GAATTCCAGGCGCG CTACTCGGTGTCC-3' ${ }^{\prime}$, antisense primer: $5^{\prime}$-TCTAGACTAGAATTTGCTACAG TCATAGAC- $3^{\prime}$ ) (DN-Foxc1: sense primer: $5^{\prime}$-GAATTCGACATGGTGAAGCC GCCCTAC- $3^{\prime}$, antisense primer: $5^{\prime}$-TCTAGATTAGATGCAGGGTGGTGGCT GGC-3'). RFP-tagged Foxc1 was generated by subcloning into pTurbo RFP-C (Evrogen, Moscow, Russia). DNA fragments of Gli2 and DN mutants of Gli2 (amino acids 2-497) were generated and tagged with the Myc-epitope by subcloning PCR products into pcDNA3 (ref. 67). Transfection of expression vectors was carried out using FuGENE6 (Roche) according to the manufacturer's protocol.

Generation of adenovirus. Recombinant adenoviruses carrying Flag-tagged cDNAs of Foxc1, Flag-tagged DN-Foxc1, Flag-tagged-F112S mutant, Flag-tagged Foxc2, Myc-tagged Gli2 and Myc-tagged DN-Gli2 (ref. 67) was constructed by homologous recombination between the pAxCAwt expression cosmid cassette (Takara Bio, Shiga, Japan) and the parental virus genome in HEK293 cells. The viruses were confirmed to retain no proliferative activity in cells other than HEK293 cells. The expression of proteins was determined by western blotting using anti-Flag antibodies.

Western blotting. Cells were rinsed twice with PBS and solubilized in lysis buffer (20 mM HEPES (pH 7.4), $150 \mathrm{mM} \mathrm{NaCl}, 1 \mathrm{mM}$ EGTA, $1.5 \mathrm{mM} \mathrm{MgCl} 2,10 \%$ glycerol, $1 \%$ Triton-X-100, $10 \mu \mathrm{g} \mathrm{ml}^{-1}$ aprotinin, $10 \mu \mathrm{g} \mathrm{ml}^{-1}$ leupeptin, $1 \mathrm{mM} \mathrm{4-(2-}$ aminoethyl) benzenesulfonyl fluoride hydrochloride, $0.2 \mathrm{mM}$ sodium orthovanadate). The lysates were centrifuged for $10 \mathrm{~min}$ at $4{ }^{\circ} \mathrm{C}$ at $15,000 \mathrm{~g}$ and boiled in SDS sample buffer containing $0.5 \mathrm{M} \beta$-mercaptoethanol for $5 \mathrm{~min}$. Samples were then separated by SDS-polyacrylamide gel electrophoresis, transferred to nitrocellulose membranes, immunoblotted with primary antibodies and visualized with horseradish peroxidase-coupled anti-mouse $(1: 10,000)$ or -rabbit $(1: 5,000)$ immunoglobulin IgG antibodies using enhanced chemiluminescence detection kits (GE Healthcare, Buckinghamshire, UK). Anti-Flag (\#F3165, 1:10,000) antibodies were purchased from Sigma-Aldrich. Anti-Myc (\#ab9132, 1:2,000) was purchased from Abcam. Full scans of western blotting data are shown in Supplementary Fig. 14.

Reporter assays. A firefly luciferase reporter construct containing six copies of the Foxc1-binding element from the PTHrP promoter (5'-CAGCAGGACTAAATAAC CAAATCCT $\left.-3^{\prime}\right)$ or from the Colloal promoter (5'-TAAAAGTATTTATTTATG TGTAAGT-3') was subcloned into pGL4.23 (Promega, WI, USA). Reporter constructs were co-transfected with Foxc1 expression vectors into COS-7 cells using FuGENE6. Forty-eight hours after transfection, the cells were lysed and luciferase activity was measured using specific substrates in a luminometer (Promega) according to the manufacturer's protocol.

IP-western analysis. Cells transfected with Flag-Foxc1 and Myc-Gli2 were washed three times with ice-cold PBS and solubilized in lysis buffer. The lysates were centrifuged at $15,000 \mathrm{~g}$ for $20 \mathrm{~min}$ at $4{ }^{\circ} \mathrm{C}$ and incubated with anti-Flag M2 magnetic beads for $4 \mathrm{~h}$ at $4{ }^{\circ} \mathrm{C}$. Immunoprecipitated samples were collected by a magnetic separator and washed five times with ice-cold PBS. Samples were boiled in SDS sample buffer and supernatants were subsequently subjected to western blot analysis.

Confocal microscopy. RFP-tagged WT or RFP-tagged F112S mutant Foxcl were concomitantly transfected with Venus-tagged Gli2 into HEK293 cells using FuGENE 6. Forty-eight hours after transfection, the cells were fixed with $4 \%$ formaldehyde/PBS and washed three times with PBS. Samples were visualized using the Nikon A1 confocal microscope system (Nikon Instech, Tokyo, Japan). The images of $x-z$ and $y-z$ stacks were obtained using Nikon NIS-Elements AR software.

DNA pull-down assays. 293FT cells were transfected with Flag-Foxc1 and lysed using lysis buffer (20 mM HEPES (pH 7.4), $150 \mathrm{mM} \mathrm{NaCl}, 1 \mathrm{mM}$ EGTA, $1.5 \mathrm{mM}$ $\mathrm{MgCl}_{2}, 10 \%$ glycerol, $1 \%$ Triton-X-100, $10 \mu \mathrm{g} \mathrm{ml}^{-1}$ leupeptin, $1 \mathrm{mM}$ phenylmethanesulfonyl fluoride, $0.2 \mathrm{mM}$ sodium orthovanadate), then incubated for $3 \mathrm{~h}$ with $1 \mu \mathrm{g}$ of a biotinylated double-stranded oligonucleotide probe containing the Foxc1-binding element present in the PTHrP promoter (sense primer: $5^{\prime}$-AGC AGGACTAAATAACCAAATCCTT- ${ }^{\prime}$, antisense primer: $5^{\prime}$-AAGGATTTGGTT ATTTAGTCCTGCT- $3^{\prime}$ ) or in the Colloal promoter (sense primer: $5^{\prime}$-AAAGCT CGAGAATATTGACTGAGATATGAAC- $3^{\prime}$, antisense primer: $5^{\prime}$-GTTCATATCT CAGTCAATATTCTCGAGCTTT- $3^{\prime}$ ). Precipitated oligonucleotides were collected using Streptavidin magnetic beads (Dynabeads My One Streptavidin T1, Invitrogen) and washed with lysis buffer. Magnetic beads were resuspended with SDS sample buffer, boiled for $5 \mathrm{~min}$ and subjected to western blot analysis.

ChIP assays. ChIP analysis was performed using a ChIP assay kit (ChIP-IT Express; Active Motif, CA, USA) according to the manufacturer's instructions. In brief, the primary chondrocytes infected with Foxcl or control adenovirus were washed with PBS, and the chromatin was fixed with $1 \%$ PFA. Chromatin was prepared and immunoprecipitated with $2 \mu \mathrm{g}$ anti-Foxc1 (\#ab5079) or anti-Gli2 (\#ab9132) antibodies (Abcam). DNA fragments were precipitated with protein-A magnetic beads and amplified by PCR using primer pairs specific to the PTHrP promoter containing the Foxcl-binding element. Quantitative analysis of ChIP assays was performed with qPCR and relevant primer pairs are listed in Supplementary Table 2.

Statistical analysis. Data were statistically analysed by Student's $t$-test to compare differences between two groups. For more than two groups, we used a one-way analysis of variance or two-way analysis of variance followed by the Tukey-Kramer test. $P$ values $<0.05$ were considered statistically significant.

\section{References}

1. Karsenty, G., Kronenberg, H. M. \& Settembre, C. Genetic control of bone formation. Annu. Rev. Cell Dev. Biol. 25, 629-648 (2009).

2. Long, F. Building strong bones: molecular regulation of the osteoblast lineage. Nat. Rev. Mol. Cell Biol. 13, 27-38 (2012).

3. Ornitz, D. M. FGF signaling in the developing endochondral skeleton. Cytokine Growth Factor Rev. 16, 205-213 (2005).

4. Kronenberg, H. M. Developmental regulation of the growth plate. Nature 423, 332-336 (2003).

5. de Crombrugghe, B. et al. Transcriptional mechanisms of chondrocyte differentiation. Matrix Biol. 19, 389-394 (2000).

6. Maes, C. Role and regulation of vascularization processes in endochondral bones. Calcif. Tissue Int. 92, 307-323 (2013).

7. Krakow, D. \& Rimoin, D. L. The skeletal dysplasias. Genet. Med. 12, 327-341 (2010).

8. Pogue, R. \& Lyons, K. BMP signaling in the cartilage growth plate. Curr. Top Dev. Biol. 76, 1-48 (2006).

9. Nishimura, R., Hata, K., Matsubara, T., Wakabayashi, M. \& Yoneda, T. Regulation of bone and cartilage development by network between BMP signalling and transcription factors. J. Biochem. 151, 247-254 (2012).

10. Retting, K. N., Song, B., Yoon, B. S. \& Lyons, K. M. BMP canonical Smad signaling through Smad1 and Smad5 is required for endochondral bone formation. Development 136, 1093-1104 (2009).

11. Amano, K. et al. MSX2 stimulates chondrocyte maturation by controlling Ihh expression. J. Biol. Chem. 283, 29513-29521 (2008).

12. Ingham, P. W. \& McMahon, A. P. Hedgehog signaling in animal development: paradigms and principles. Genes Dev. 15, 3059-3087 (2001).

13. Lai, L. P. \& Mitchell, J. Indian hedgehog: its roles and regulation in endochondral bone development. J. Cell Biochem. 96, 1163-1173 (2005).

14. Mak, K. K., Kronenberg, H. M., Chuang, P. T., Mackem, S. \& Yang, Y. Indian hedgehog signals independently of PTHrP to promote chondrocyte hypertrophy. Development 135, 1947-1956 (2008).

15. St-Jacques, B., Hammerschmidt, M. \& McMahon, A. P. Indian hedgehog signaling regulates proliferation and differentiation of chondrocytes and is essential for bone formation. Genes Dev. 13, 2072-2086 (1999).

16. Kesper, D. A., Didt-Koziel, L. \& Vortkamp, A. Gli2 activator function in preosteoblasts is sufficient to mediate Ihh-dependent osteoblast differentiation, whereas the repressor function of Gli2 is dispensable for endochondral ossification. Dev. Dyn. 239, 1818-1826 (2010).

17. Miao, D. et al. Impaired endochondral bone development and osteopenia in Gli2-deficient mice. Exp. Cell Res. 294, 210-222 (2004).

18. Akiyama, H., Chaboissier, M. C., Martin, J. F., Schedl, A. \& de Crombrugghe, B. The transcription factor Sox9 has essential roles in successive steps of the 
chondrocyte differentiation pathway and is required for expression of Sox 5 and Sox6. Genes Dev. 16, 2813-2828 (2002).

19. Yoshida, C. A. et al. Runx2 and Runx 3 are essential for chondrocyte maturation, and Runx2 regulates limb growth through induction of Indian hedgehog. Genes Dev. 18, 952-963 (2004).

20. Wuelling, M. \& Vortkamp, A. Transcriptional networks controlling chondrocyte proliferation and differentiation during endochondral ossification. Pediatr. Nephrol. 25, 625-631 (2010).

21. Amano, K. et al. Arid5a cooperates with Sox9 to stimulate chondrocyte-specific transcription. Mol. Biol. Cell 22, 1300-1311 (2011).

22. Hata, K. et al. Paraspeckle protein p54nrb links Sox9-mediated transcription with RNA processing during chondrogenesis in mice. J. Clin. Invest. 118, 3098-3108 (2008).

23. Muramatsu, S. et al. Functional gene screening system identified TRPV4 as a regulator of chondrogenic differentiation. J. Biol. Chem. 282, 32158-32167 (2007).

24. Takigawa, Y. et al. The transcription factor Znf219 regulates chondrocyte differentiation by assembling a transcription factory with Sox9. J. Cell Sci. 123, 3780-3788 (2010).

25. Hata, K. et al. Arid5b facilitates chondrogenesis by recruiting the histone demethylase Phf2 to Sox9-regulated genes. Nat. Commun. 4, 2850 (2013).

26. Nishimura, D. Y. et al. The forkhead transcription factor gene FKHL7 is responsible for glaucoma phenotypes which map to 6p25. Nat. Genet. 19, 140-147 (1998).

27. Tumer, Z. \& Bach-Holm, D. Axenfeld-Rieger syndrome and spectrum of PITX2 and FOXC1 mutations. Eur. J. Hum. Genet. 17, 1527-1539 (2009).

28. Hong, H. K., Lass, J. H. \& Chakravarti, A. Pleiotropic skeletal and ocular phenotypes of the mouse mutation congenital hydrocephalus (ch/Mf1) arise from a winged helix/forkhead transcriptionfactor gene. Hum. Mol. Genet. 8, 625-637 (1999)

29. Saleem, R. A., Banerjee-Basu, S., Murphy, T. C., Baxevanis, A. \& Walter, M. A. Essential structural and functional determinants within the forkhead domain of FOXC1. Nucleic Acids Res. 32, $4182-4193$ (2004).

30. Vortkamp, A. et al. Regulation of rate of cartilage differentiation by Indian hedgehog and PTH-related protein. Science 273, 613-622 (1996).

31. Ikram, M. S. et al. GLI2 is expressed in normal human epidermis and BCC and induces GLI1 expression by binding to its promoter. J. Invest. Dermatol. 122, 1503-1509 (2004).

32. Agren, M., Kogerman, P., Kleman, M. I., Wessling, M. \& Toftgard, R. Expression of the PTCH1 tumor suppressor gene is regulated by alternative promoters and a single functional Gli-binding site. Gene 330, 101-114 (2004).

33. Berry, F. B., Saleem, R. A. \& Walter, M. A. FOXC1 transcriptional regulation is mediated by $\mathrm{N}$ - and $\mathrm{C}$-terminal activation domains and contains a phosphorylated transcriptional inhibitory domain. J. Biol. Chem. 277, 10292-10297 (2002).

34. Amizuka, N., Warshawsky, H., Henderson, J. E., Goltzman, D. \& Karaplis, A. C. Parathyroid hormone-related peptide-depleted mice show abnormal epiphyseal cartilage development and altered endochondral bone formation. J. Cell Biol. 126, 1611-1623 (1994).

35. Karaplis, A. C. et al. Lethal skeletal dysplasia from targeted disruption of the parathyroid hormone-related peptide gene. Genes Dev. 8, 277-289 (1994).

36. Hannenhalli, S. \& Kaestner, K. H. The evolution of Fox genes and their role in development and disease. Nat. Rev. Genet. 10, 233-240 (2009).

37. Nifuji, A., Miura, N., Kato, N., Kellermann, O. \& Noda, M. Bone morphogenetic protein regulation of forkhead/winged helix transcription factor Foxc2 (Mfh1) in a murine mesodermal cell line $\mathrm{C} 1$ and in skeletal precursor cells. J. Bone Miner. Res. 16, 1765-1771 (2001).

38. Chang, T. C., Summers, C. G., Schimmenti, L. A. \& Grajewski, A. L. AxenfeldRieger syndrome: new perspectives. Br. J. Ophthalmol. 96, 318-322 (2012).

39. Kannu, P., Oei, P., Slater, H. R., Khammy, O. \& Aftimos, S. Epiphyseal dysplasia and other skeletal anomalies in a patient with the $6 \mathrm{p} 25$ microdeletion syndrome. Am. J. Med. Genet. A 140, 1955-1959 (2006).

40. Klopocki, E. et al. Deletion and point mutations of PTHLH cause brachydactyly type E. Am. J. Hum. Genet. 86, 434-439 (2010).

41. Honkanen, R. A. et al. A family with Axenfeld-Rieger syndrome and Peters Anomaly caused by a point mutation (Phe112Ser) in the FOXC1 gene. Am. J. Ophthalmol. 135, 368-375 (2003).

42. Kume, T., Jiang, H., Topczewska, J. M. \& Hogan, B. L. The murine winged helix transcription factors, Foxc1 and Foxc2, are both required for cardiovascular development and somitogenesis. Genes Dev. 15, 2470-2482 (2001).

43. Kume, T., Deng, K. \& Hogan, B. L. Murine forkhead/winged helix genes Foxc1 (Mf1) and Foxc2 (Mfh1) are required for the early organogenesis of the kidney and urinary tract. Development 127, 1387-1395 (2000).

44. Kidson, S. H., Kume, T., Deng, K., Winfrey, V. \& Hogan, B. L. The forkhead/ winged-helix gene, Mfl, is necessary for the normal development of the cornea and formation of the anterior chamber in the mouse eye. Dev. Biol. 211, 306-322 (1999).

45. Kume, T. et al. The forkhead/winged helix gene Mf1 is disrupted in the pleiotropic mouse mutation congenital hydrocephalus. Cell 93, 985-996 (1998).
46. Rice, R., Rice, D. P. \& Thesleff, I. Foxc1 integrates Fgf and Bmp signalling independently of twist or noggin during calvarial bone development. Dev. Dyn. 233, 847-852 (2005).

47. Rice, R., Rice, D. P., Olsen, B. R. \& Thesleff, I. Progression of calvarial bone development requires Foxc1 regulation of Msx2 and Alx4. Dev. Biol. 262, 75-87 (2003).

48. Mirzayans, F., Lavy, R., Penner-Chea, J. \& Berry, F. B. Initiation of early osteoblast differentiation events through the direct transcriptional regulation of Msx2 by FOXC1. PLoS ONE 7, e49095 (2012).

49. Mo, R. et al. Specific and redundant functions of Gli2 and Gli3 zinc finger genes in skeletal patterning and development. Development 124, 113-123 (1997).

50. Nakamura, Y. et al. Wwp2 is essential for palatogenesis mediated by the interaction between Sox9 and mediator subunit 25. Nat. Commun. 2, 251 (2011).

51. Ieda, M. et al. Direct reprogramming of fibroblasts into functional cardiomyocytes by defined factors. Cell 142, 375-386 (2010).

52. Lalmansingh, A. S., Karmakar, S., Jin, Y. \& Nagaich, A. K. Multiple modes of chromatin remodeling by Forkhead box proteins. Biochim. Biophys. Acta 1819, 707-715 (2012).

53. Wang, Q. et al. A hierarchical network of transcription factors governs androgen receptor-dependent prostate cancer growth. Mol. Cell 27, 380-392 (2007).

54. Carroll, J. S. et al. Chromosome-wide mapping of estrogen receptor binding reveals long-range regulation requiring the forkhead protein FoxA1. Cell 122, 33-43 (2005).

55. Kimura, A. et al. Runx1 and Runx2 cooperate during sternal morphogenesis. Development 137, 1159-1167 (2010).

56. Mattiske, D., Kume, T. \& Hogan, B. L. The mouse forkhead gene Foxc1 is required for primordial germ cell migration and antral follicle development. Dev. Biol. 290, 447-458 (2006)

57. Hayashi, H. \& Kume, T. Forkhead transcription factors regulate expression of the chemokine receptor CXCR4 in endothelial cells and CXCL12-induced cell migration. Biochem. Biophys. Res. Commun. 367, 584-589 (2008).

58. Ray, P. S. et al. FOXC1 is a potential prognostic biomarker with functional significance in basal-like breast cancer. Cancer Res. 70, 3870-3876 (2010).

59. Iida, K. et al. Essential roles of the winged helix transcription factor MFH-1 in aortic arch patterning and skeletogenesis. Development 124, 4627-4638 (1997).

60. Saleem, R. A., Banerjee-Basu, S., Berry, F. B., Baxevanis, A. D. \& Walter, M. A. Analyses of the effects that disease-causing missense mutations have on the structure and function of the winged-helix protein FOXC1. Am. J. Hum. Genet. 68, 627-641 (2001).

61. Saleem, R. A., Banerjee-Basu, S., Berry, F. B., Baxevanis, A. D. \& Walter, M. A. Structural and functional analyses of disease-causing missense mutations in the forkhead domain of FOXC1. Hum. Mol. Genet. 12, 2993-3005 (2003).

62. Beck, F., Tucci, J., Russell, A., Senior, P. V. \& Ferguson, M. W. The expression of the gene coding for parathyroid hormone-related protein (PTHrP) during tooth development in the rat. Cell Tissue Res. 280, 283-290 (1995).

63. Lee, K., Deeds, J. D. \& Segre, G. V. Expression of parathyroid hormone-related peptide and its receptor messenger ribonucleic acids during fetal development of rats. Endocrinology 136, 453-463 (1995).

64. Schipani, E. et al. Targeted expression of constitutively active receptors for parathyroid hormone and parathyroid hormone-related peptide delays endochondral bone formation and rescues mice that lack parathyroid hormone-related peptide. Proc. Natl Acad. Sci. USA 94, 13689-13694 (1997).

65. Vivatbutsiri, P. et al. Impaired meningeal development in association with apical expansion of calvarial bone osteogenesis in the Foxcl mutant. J. Anat. 212, 603-611 (2008).

66. Gartland, A. et al. In vitro chondrocyte differentiation using costochondral chondrocytes as a source of primary rat chondrocyte cultures: an improved isolation and cryopreservation method. Bone 37, 530-544 (2005).

67. Shimoyama, A. et al. Ihh/Gli2 signaling promotes osteoblast differentiation by regulating Runx2 expression and function. Mol. Biol. Cell 18, 2411-2418 (2007).

\section{Acknowledgements}

We thank Benoit de Crombrugghe (The University of Texas, MD Anderson Cancer Center) for the Col2al gene promoter and Atsushi Miyawaki (RIKEN, BSI) for the Venus construct. We are grateful to Kirsi Sainio (University of Helsinki) for providing Foxc1 ${ }^{\text {ch }}$ $c h$ mice. This work was supported in part by the Japanese Ministry of Education, Culture, Sports, Science and Technology Grants-in-Aid for Scientific Research (K.H. and R.N.), and 'Challenge to Intractable Oral Diseases' (K.H.), the Uehara Memorial Foundation (K.H. and R.N.), the Senri Life Science Foundation (K.H.), the Nakatomi Foundation (K.H.), and the Mochida Memorial Foundation for Medical and Pharmaceutical Research (K.H.) 


\section{Author contributions}

K.H and R.N. directed the study. K.H. and M.Y. designed and performed all the in vitro and in vivo experiments. R.T., E.N., K.O., Y.T. and T.M. performed the molecular and biochemical experiments. S.I., T.T.-Y., and T.Y. discussed and assessed the data. K.H., M.Y. and R.N. wrote the paper.

\section{Additional information}

Accession codes: Microarray data have been deposited in the NCBI Gene Expression Omnibus (http://www.ncbi.nlm.nih.gov/geo/) under the accession code GSE65350.
Supplementary Information accompanies this paper at http://www.nature.com/ naturecommunications

Competing financial interests: There are no competing financial interests.

Reprints and permission information is available online at http://npg.nature.com/ reprintsandpermissions/

How to cite this article: Yoshida, M. et al. The transcription factor Foxcl is necessary for Ihh-Gli2-regulated endochondral ossification. Nat. Commun. 6:6653 doi: $10.1038 /$ ncomms7653 (2015). 